\title{
REAL TROPICALIZATION AND ANALYTIFICATION OF SEMIALGEBRAIC SETS
}

\author{
PHILIPP JELL, CLAUS SCHEIDERER, AND JOSEPHINE YU
}

\begin{abstract}
Let $K$ be a real closed field with a nontrivial non-archimedean absolute value. We study a refined version of the tropicalization map, which we call real tropicalization map, that takes into account the signs on $K$. We study images of semialgebraic subsets of $K^{n}$ under this map from a general point of view. For a semialgebraic set $S \subseteq K^{n}$ we define a space $S_{r}^{\text {an }}$ called the real analytification, which we show to be homeomorphic to the inverse limit of all real tropicalizations of $S$. We prove a real analogue of the tropical fundamental theorem and show that the tropicalization of any semialgebraic set is described by tropicalization of finitely many inequalities which are valid on the semialgebraic set. We also study the topological properties of real analytification and tropicalization. If $X$ is an algebraic variety, we show that $X_{r}^{\text {an }}$ can be canonically embedded into the real spectrum $X_{r}$ of $X$, and we study its relation with the Berkovich analytification of $X$.
\end{abstract}

\section{INTRODUCTION}

Let $K$ be an algebraically closed field which is equipped with a non-archimedean nontrivial absolute value $|$.$| . The map$

$$
\text { trop: } K^{n} \rightarrow(\mathbb{R} \cup\{-\infty\})^{n}, \quad\left(x_{1}, \ldots, x_{n}\right) \mapsto\left(\log \left|x_{1}\right|, \ldots, \log \left|x_{n}\right|\right)
$$

is called the tropicalization map. For a closed algebraic subvariety $X$ of $K^{n}$, the associated tropical variety $\operatorname{Trop}(X)$ is defined as the closure in $(\mathbb{R} \cup\{-\infty\})^{n}$ of $\operatorname{trop}(X)$. In good situations, this tropical variety contains information about $X$, such as its dimension, degree, Chow cohomology class [MS15, §6.7] or the valuation of the $j$-invariant when $X$ is an elliptic curve KMM09.

Given a variety $X$ over $K$, Berkovich defines the analytification $X^{\text {an }}$ of $X$, which is a connected Hausdorff topological space that contains $X(K)$ as a dense subset Ber90. If $X$ is a closed subvariety of $\mathbb{A}^{n}$, the tropicalization map above naturally extends to $X^{\text {an }}$. The fundamental theorem of tropical geometry states that $\operatorname{Trop}(X)=\operatorname{trop}\left(X^{\text {an }}\right)$ and that $\operatorname{Trop}(X)$ can be characterized by tropicalizing the polynomials that vanish on $X$ (see [Dra08, Theorem 4.2], [EKL06, [MS15, §3.2]). Given an affine variety over $K$, Payne shows that $X^{\text {an }}$ is homeomorphic to the inverse limit of tropicalizations of all embeddings of $X$ into affine spaces Pay09, Theorem 1.1].

In this paper, we consider a similar setup, but with the following modifications and generalizations:

2010 Mathematics Subject Classification. Primary 14P10, secondary 14T05, 14G22, 32P05.

PJ was supported by the DFG Research Fellowship JE 856/1-1, CS was supported in part by DFG grant SCHE281/10-2, and JY was supported in part by NSF grant DMS-1600569. 
(1) $K$ is real closed instead of algebraically closed;

(2) the tropicalization takes the order on $K$ into account;

(3) $X$ does not need to be an algebraic variety but may be only a semialgebraic set.

Our goal is to define a space $X_{r}^{\text {an }}$, which we call real analytification of $X$, and prove analogues of the fundamental theorem and the limit theorem for it.

For an algebraic variety we define $X_{r}^{\text {an }}$ in Definition 3.1 and for a semialgebraic set $S \subseteq K^{n}$, we define $S_{r}^{\text {an }}$ in Definition 4.1. We prove an analogue of the tropical fundamental theorem in Theorem 6.9 and the analogue of Payne's theorem in Theorem 6.14.

Let us provide some more details. Let $K$ be a real closed field with a nontrivial nonarchimedean absolute value $|\cdot|_{K}$ that is compatible with the order on $K$. An example is the field of real Puiseux series $\mathbb{R}\{\{t\}\}=\bigcup_{n \in \mathbb{N}>0} \mathbb{R}\left(\left(t^{1 / n}\right)\right)$, where the positive Puiseux series are those with positive leading coefficients. The real tropicalization map is defined as

$$
\operatorname{trop}_{r}: K^{n} \rightarrow \mathbb{R}^{n}, \quad\left(x_{1}, \ldots, x_{n}\right) \mapsto\left(\operatorname{sgn}\left(x_{1}\right)\left|x_{1}\right|_{K}, \ldots, \operatorname{sgn}\left(x_{n}\right)\left|x_{n}\right|_{K}\right) .
$$

For a semialgebraic set $S \subseteq K^{n}$, the real tropicalization $\operatorname{Trop}_{r}(S)$ is defined to be the

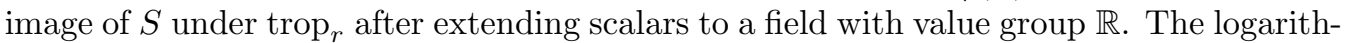
mic version of this construction, without signs, was first used by Alessandrini in [Ale13. For polytopes this was done earlier in [DY07. We do not know of general algorithms for computing tropicalizations of real varieties or semialgebraic sets, but there are some descriptions of tropicalized spectrahedra [AGS16] and numerical methods in the case of curves defined over $\mathbb{R}$ BHV19.

Since we do not want to restrict to a fixed orthant, we choose to omit the logarithm to simplify notation and to make the topology more apparent. This means that our $\operatorname{Trop}_{r}(S)$ is not piecewise linear, but it is more meaningful topologically since we are taking into account all orthants, similarly to Viro's patchworking for hypersurfaces Vir84. For example, Celaya showed recently that the real tropicalization of a tropical linear space defined over $\mathbb{R}$, and more generally the real Bergman fan of an oriented matroid, is the cone over a piecewise linear topological sphere in $\mathbb{R}^{n}$ [Cel18. We will see in Corollary 6.10 that if $S$ is semialgebraically connected, then its real tropicalization is connected.

For an affine $K$-variety $X=\operatorname{Spec}(A)$, the Berkovich analytification $X^{\text {an }}$ of $X$ consists of the $K$-seminorms on $A$, and the real spectrum $X_{r}$ consists of the orderings on $A$. We will review these notions in more detail in $\$ 2$. In $\$ 3$ we define the real analytification $X_{r}^{\text {an }}$ as a topological space consisting of signed seminorms on the coordinate ring. We also introduce $X_{r}^{\text {an }}$ for non-affine varieties. We study the topological properties of $X_{r}^{\text {an }}$ and its canonical maps $X_{r}^{\text {an }} \rightarrow X^{\text {an }}$ and $X_{r}^{\text {an }} \rightarrow X_{r}$ in $\$ 3$ In comparison with the real spectrum $X_{r}$, we will see that the real analytification $X_{r}^{\text {an }}$ is in fact homeomorphic to a subspace of $X_{r}$ which can be described entirely in terms of orders, consisting of the so-called relatively archimedean points.

In $\$ 4$ we study the real analytification $S_{r}^{\text {an }}$ for a semialgebraic set $S$. In $\$$ we explain how to tropicalize polynomial inequalities. To do this, we take a quick excursion into the realm of hyperfields. Hyperfields are algebraic structures similar to fields, but which allow multivalued addition. Jun shows in Jun17, that both the Berkovich space $X^{\text {an }}$ and the real spectrum $X_{r}$ of a variety can be seen as the set of points of $X$ over the tropical hyperfield and the sign hyperfield respectively. We recall his results and show that $X_{r}^{\text {an }}$ 
can similarly be seen as the set of points of $X$ over the tropical real hyperfield. The results by Jun provided some of our inspiration for the definition of $X_{r}^{\text {an }}$ and the tropicalization of polynomial inequalities.

In $₫ 6$ we turn our attention to the real tropicalization map and prove our two main theorems 6.9 and 6.14 mentioned above. An important step is Theorem 6.2, which shows that any point in $\operatorname{trop}_{r}\left(S_{r}^{\text {an }}\right)$ that satisfies an obviously necessary condition is already in $\operatorname{trop}_{r}(S)$. We also prove the existence of a finite tropical description for semialgebraic sets in each orthant.

\section{BACKGROUND}

For a commutative ring $A$, a subset $P \subseteq A$ is called an ordering of $A$ if $P+P \subseteq P$, $P \cdot P \subseteq P, P \cup-P=A$, and $P \cap-P$ is a prime ideal of $A$. The support of $P$ is $\operatorname{supp}(P):=P \cap-P$. With $P$ we associate an order relation and a sign function in the natural way: Write $f>_{P} 0$ and $\operatorname{sgn}_{P}(f)=+1$ if $-f \notin P$, write $f \geq_{P} 0$ if $f \in P$ and $\operatorname{sgn}_{P}(f)=0$ if $f \in \operatorname{supp}(P)$. We can define $<_{P}$ and $\leq_{P}$ in the obvious way, and we have $\operatorname{sgn}_{P}(-f)=-\operatorname{sgn}_{P}(f)$. We may refer to the ordering as either $P$ or $<_{P}$.

The real spectrum $\operatorname{Sper}(A)$ of a commutative ring $A$ is the set of orderings of $A$, with the Harrison topology given by the subbasis of open sets $\left\{P \in \operatorname{Sper}(A): f>_{P} 0\right\}$ for $f \in A$. See [BCR98] or [KS89, for example. For an affine scheme $X=\operatorname{Spec}(A)$, the real spectrum $X_{r}$ is defined to be $\operatorname{Sper}(A)$. The real spectrum $X_{r}$ of an arbitrary scheme $X$ is defined by glueing the real spectra of open affine subspaces. A point in $X_{r}$ is a pair $(p, P)$ where $p$ is a scheme point of $X$ and $P$ is an ordering of the residue field $K(p)$ of $p$.

For example, for $X=\mathbb{A}_{\mathbb{R}}^{1}$, the orderings on $A=\mathbb{R}[T]$ are classified as follows (see, for example, [BCR98, Example 7.1.4):

$$
\begin{aligned}
P_{a} & =\{f(T) \in \mathbb{R}[T]: f(a) \geq 0\}, \text { for } a \in \mathbb{R} \\
P_{a^{+}} & =\{f(T) \in \mathbb{R}[T]: f \geq 0 \text { on some interval }[a, a+\varepsilon], \varepsilon>0\}, \text { for } a \in \mathbb{R} \\
P_{a^{-}} & =\{f(T) \in \mathbb{R}[T]: f \geq 0 \text { on some interval }[a-\varepsilon, a], \varepsilon>0\}, \text { for } a \in \mathbb{R} \\
P_{\infty} & =\{f(T) \in \mathbb{R}[T]: f \geq 0 \text { on some interval }[b, \infty[\text { with } b \in \mathbb{R}\} \\
P_{-\infty} & =\{f(T) \in \mathbb{R}[T]: f \geq 0 \text { on some interval }]-\infty, b] \text { with } b \in \mathbb{R}\}
\end{aligned}
$$

For $P_{a}$, the support is $\langle T-a\rangle$, and the residue field is $\mathbb{R}$, which has a unique order. The other four types have support $\{0\}$ with residue field $\mathbb{R}(T)$. The real spectrum $\operatorname{Sper} \mathbb{R}(T)$ is identified with the subspace of Sper $\mathbb{R}[T]$ consisting of orderings of the form $P_{a^{+}}, P_{a^{-}}, P_{\infty}$ and $P_{-\infty}$. We can think of $P_{a^{+}}$(resp. $P_{a^{-}}$) as orders in which $T$ is infinitesimally larger (resp. smaller) than $a$. The points of the form $P_{a}, P_{\infty}, P_{-\infty}$ are closed, while $\overline{\left\{P_{a^{+}}\right\}}=\left\{P_{a^{+}}, P_{a}\right\}$ and $\overline{\left\{P_{a^{-}}\right\}}=\left\{P_{a^{-}}, P_{a}\right\}$.

Let $K$ denote a real closed field with a nontrivial non-archimedean valuation $\nu: K^{*} \rightarrow$ $\mathbb{R}$, giving a non-archimedean absolute value $|a|_{K}=|a|=e^{-\nu(a)}\left(a \in K^{*}\right),|0|=0$. Being real closed, $K$ has a unique ordering. We will always assume that the absolute value is compatible with this ordering: $0 \leq a \leq b \Rightarrow|a|_{K} \leq|b|_{K}$, or equivalently, $|a|_{K}>|b|_{K} \Rightarrow \operatorname{sgn}(a+b)=\operatorname{sgn}(a)=\operatorname{sgn}(a-b)$. An example is the Puiseux series field $K=\mathbb{R}\{\{t\}\}=\bigcup_{n \geq 0} \mathbb{R}\left(\left(t^{\frac{1}{n}}\right)\right)$. We will equip $K$ with the topology induced by the order. The compatibility ensures that this topology is coarser then the one induced by 
the non-archimedean absolute value and both topologies agree if the absolute value is non-trivial.

For a $K$-algebra $A$, a (non-archimedean multiplicative) $K$-seminorm on $A$ is a map $|\cdot|_{x}: A \rightarrow \mathbb{R}_{\geq 0}$ such that $|a|_{x}=|a|_{K}$ for $a \in K$ and $|f g|_{x}=|f|_{x} \cdot|g|_{x}$, and $|f+g|_{x} \leq$ $\max \left(|f|_{x},|g|_{x}\right)(f, g \in A)$. For this text we only work with non-archimedean multiplicative $K$-seminorms and simply write " $K$-seminorm". The support of the seminorm $|\cdot|_{x}$ is the prime ideal $\operatorname{supp}\left(|\cdot|_{x}\right)=\left\{f \in A:|f|_{x}=0\right\}$. An absolute value is a seminorm whose support is $\{0\}$.

The Berkovich spectrum $\mathcal{M}(A)$ is the set of $K$-seminorms on $A$ with the coarsest topology that makes the map $|\cdot|_{x} \mapsto|f|_{x}$ continuous for every $f \in A$. See [Ber90]. A subbasis of open sets is given by the $\left\{|{ }|_{x} \in \mathcal{M}(A): r<|f|_{x}<s\right\}$ where $r<s$ are real numbers and $f \in A$. For an affine $K$-scheme $X=\operatorname{Spec}(A)$, the analytification $X^{\text {an }}$ is defined to be $\mathcal{M}(A)$. The analytification $X^{\text {an }}$ of an arbitrary $K$-scheme $X$ is defined by glueing the spectra of open affine subschemes. A point in $X^{\text {an }}$ is a tuple $(p,||$.$) where p$ is a point in the scheme $X$ and $|$.$| is an absolute value on the residue field K(p)$ which is compatible with $|.|_{K}$ on $K$. See [Bak08] for a visualization of $\mathbb{A}^{1, \text { an }}$, which will be discussed in Example 3.12

Given any non-archimedean valued field $\left(L,|\cdot|_{L}\right)$, we denote by $k_{L}$ the residue field of the valuation ring of $L$ and by $\bar{f} \in k_{L}$ the reduction of $f \in L$ with $|f| \leq 1$. We also denote by $\Gamma_{L}:=\left|L^{*}\right|_{L}$ the value group of $L$, a multiplicative subgroup of $\mathbb{R}_{>0}$. Let $P$ be an ordering of $L$ that is compatible with $|.|_{L}$. Then $P$ induces an ordering $\bar{P}$ of the residue field $k_{L}$ by

$$
\bar{P}=\left\{\bar{a}: a \in P,|a|_{L} \leq 1\right\} .
$$

Conversely, the Baer-Krull theorem tells which orderings $P$ induce the same ordering $\bar{P}$ on $k_{L}$ :

Theorem 2.1 (Baer-Krull Theorem). Let $\left(L,|\cdot|_{L}\right)$ be a non-archimedean valued field with value group $\Gamma_{L}$. Let $\left(f_{i}\right)_{i \in I}$ be a family of elements in $L^{*}$ such that $\left(\left|f_{i}\right|_{L}\right)_{i \in I}$ is $a \mathbb{Z} / 2 \mathbb{Z}$-basis of $\Gamma_{L} / 2 \Gamma_{L}$. Given any ordering $Q$ of $k_{L}$ and any tuple $\left(\epsilon_{i}\right)_{i \in I}$ in $\{ \pm 1\}^{I}$, there is a unique ordering $P$ of $L$ that is compatible with $|\cdot|_{L}$ and satisfies $\bar{P}=Q$ and $\operatorname{sgn}_{P}\left(f_{i}\right)=\epsilon_{i}(i \in I)$. In short, the map

$$
\begin{aligned}
\left\{P \in \operatorname{Sper}(L): P \text { is compatible with }|\cdot|_{L}\right\} & \rightarrow\{-1,1\}^{I} \times \operatorname{Sper}\left(k_{L}\right), \\
P & \mapsto\left(\left(\operatorname{sgn}_{P}\left(f_{i}\right)\right)_{i \in I}, \bar{P}\right)
\end{aligned}
$$

is a bijection.

It can easily be shown that an order on a non-archimedean valued field $L$ that is compatible with $|\cdot|_{L}$ extends in a unique way to the completion $\hat{L}$ of $L$.

Definition 2.2. Let $X=\operatorname{Spec}(A)$ be an affine $K$-variety. A semialgebraic set $S \subseteq X(K)$ is a finite boolean combination of sets of the form $\{x \in X(K): f(x)>0\}$, where $f \in A$. An explicit expression for $S$ in this way is called semialgebraic description of $S$.

\section{The Real Analytification $X_{r}^{\text {an }}$}

Let $K$ be a real closed field with an order-compatible non-archimedean absolute value. We will assume that this absolute value is non-trivial in Sections 3.3 and 3.4 Let $X$ be a 
variety over $K$, by which we always mean reduced, irreducible, separated scheme of finite type. For a point $p$ in the scheme $X$, let $K(p)$ denote its residue field.

Definition 3.1. The real analytification of $X$ is the set $X_{r}^{\text {an }}$ consisting of all triples $x=\left(p_{x},|\cdot|_{x},<_{x}\right)$ where $p_{x} \in X,|\cdot|_{x}$ is an absolute value on $K\left(p_{x}\right)$ extending $|\cdot|_{K}$, and $<_{x}$ is an order on $K\left(p_{x}\right)$ compatible with $|\cdot|_{x}$. We equip $X_{r}^{\text {an }}$ with the coarsest topology such that the support map

$$
X_{r}^{\mathrm{an}} \rightarrow X, \quad x \mapsto \operatorname{supp}(x):=p_{x}
$$

is continuous and the map

$$
\operatorname{supp}^{-1}(U) \rightarrow \mathbb{R}, \quad x \mapsto \operatorname{sgn}_{x}(f) \cdot|f|_{x}=:|f|_{x}^{\text {sgn }}
$$

is continuous for every open $U \subseteq X$ and every regular functions $f$ on $U$.

Remark 3.2. Note that the real analytification $X_{r}^{\text {an }}$ is functorial in $X$ in the obvious way.

For an affine $K$-variety $X=\operatorname{Spec}(A)$, the real analytification $X_{r}^{\text {an }}$ has a description as the space of signed seminorms, which combines the constructions of $\operatorname{Sper}(A)$ and $\mathcal{M}(A)$, as we will now see.

Definition 3.3. Let $A$ be a $K$-algebra. A signed $K$-seminorm on $A$ is a map $|.|^{\mathrm{sgn}}: A \rightarrow$ $\mathbb{R}$ satisfying

(1) $|f|^{\operatorname{sgn}}=\operatorname{sgn}(f) \cdot|f|_{K}$ if $f \in K$,

(2) $|f \cdot g|^{\operatorname{sgn}}=|f|^{\text {sgn }} \cdot|g|^{\text {sgn }}$,

(3) $\min \left(|f|^{\mathrm{sgn}},|g|^{\mathrm{sgn}}\right) \leq|f+g|^{\mathrm{sgn}} \leq \max \left(|f|^{\mathrm{sgn}},|g|^{\operatorname{sgn}}\right)$

for all $f, g \in A$. The real Berkovich spectrum $\mathcal{M}_{r}(A)$ is the set of all signed $K$-seminorms on $A$, with the coarsest topology that makes the map

$$
\mathcal{M}_{r}(A) \rightarrow \mathbb{R}, \quad|\cdot|^{\text {sgn }} \mapsto|f|^{\text {sgn }}
$$

continuous for every $f$ in $A$.

Proposition 3.4. Let $A$ be a finitely generated $K$-algebra and $X=\operatorname{Spec}(A)$. Then

$$
X_{r}^{\text {an }}=\mathcal{M}_{r}(A) .
$$

Proof. Given $x=\left(p_{x},|\cdot|_{x},<_{x}\right) \in X_{r}^{\text {an }}$ we get a signed seminorm $|.|{ }_{x}^{\text {sgn }}$ on $A$ by

$$
|f|_{x}^{\text {sgn }}:=\operatorname{sgn}_{x}\left(f_{x}\right) \cdot\left|f_{x}\right|_{x}
$$

where $f_{x}=f+p_{x} \in K\left(p_{x}\right)$.

Conversely, given $|.|_{x}^{\text {sgn }} \in \mathcal{M}_{r}(A)$, we get a $K$-seminorm $|.|_{x}$ on $A$ defined by $|f|_{x}:=$ $\left.|| f\right|_{x} ^{\operatorname{sgn}} \mid$, and an ordering $\left\{f \in A:|f|_{x}^{\text {sgn }} \geq 0\right\}$, where the support of the seminorm and the support of the ordering coincide.

These procedures are mutual inverses, and it is easy to see that the topologies agree.

Proposition 3.5. Let $K$ be a real closed field with a compatible non-archimedean absolute value. Then the completion $\hat{K}$ of $K$ is also a real closed field with a compatible absolute value. Further, if $X$ is a variety over $K$, then $(X \otimes \hat{K})_{r}^{\text {an }}$ is homeomorphic to $X_{r}^{\text {an }}$. 
Proof. Let $K^{\prime}$ be the algebraic closure of $K$ and $\hat{K}^{\prime}$ its completion. Then $\hat{K}^{\prime}$ is algebraically closed and $2=\left[K^{\prime}: K\right] \geq\left[\hat{K}^{\prime}: \hat{K}\right]$. Since the order on $K$ extends to the order on $\hat{K}$, we have that $\hat{K}$ is real, thus real closed.

For the second statement, we may assume that $X$ is affine. Then the statement is equivalent to showing that any signed seminorm on a $K$-algebra $A$ extends uniquely to $A \otimes_{K} \hat{K}$.

Since a signed seminorm on $K\left[T_{1}, \ldots, T_{n}\right] / \mathfrak{a}$ where $\mathfrak{a}$ is an ideal is just a signed seminorm on $K\left[T_{1}, \ldots, T_{n}\right]$ whose associated prime contains $\mathfrak{a}$, we may reduce to the case $X=\mathbb{A}^{n}$.

We use the map $\left(\mathbb{A}_{\hat{K}}^{n}\right)_{r}^{\text {an }} \rightarrow\left(\mathbb{A}_{K}^{n}\right)_{r}^{\text {an }}$ given by restriction of signed seminorms. We construct a section to show that this map is bijective. We will use the multiindex notation; that is, for a tuple $I=\left(i_{1}, \ldots, i_{n}\right)$, we write $T^{I}:=\prod_{j=1}^{n} T^{i_{j}}$ and $|I|=\sum_{j=1}^{n} i_{j}$. Given a signed seminorm $|.|^{\operatorname{sgn}}$ on $K\left[T_{1}, \ldots, T_{n}\right]$ and $f=\sum_{I:|I| \leq d} a_{I} T^{I} \in \hat{K}\left[T_{1}, \ldots, T_{n}\right]$ we pick sequences $b_{I, j} \rightarrow a_{I}$ and define $f_{j}:=\sum_{I:|I| \leq d} b_{I, j} T^{I}$ and $|f|^{\mathrm{sgn}}=\lim _{j \rightarrow \infty}\left|f_{j}\right|^{\mathrm{sgn}}$. It is easy to check that this is well defined and indeed defines an extension of $|.|^{\text {sgn }}$.

We now identify these two sets along this bijection and consider the two topologies, one defined by $\left(\mathbb{A}_{K}^{n}\right)_{r}^{\text {an }}$ and the other one by $\left(\mathbb{A}_{\hat{K}}^{n}\right)_{r}^{\text {an }}$. Clearly the one defined by $\hat{K}$ is finer then the one defined by $K$.

To see that the topologies agree, fix $f=\sum_{I:|I|<d} a_{I} T^{I} \in \hat{K}\left[T_{1}, \ldots, T_{n}\right]$ and $c \in \mathbb{R}$. Take $|\cdot|_{y}^{\mathrm{sgn}} \in\left(\mathbb{A}_{K}^{n}\right)_{r}^{\text {an }}$ with $|f|_{y}^{\mathrm{sgn}}>c$ and fix $s>\left|T_{i}\right|_{y}$ for all $i$. Take $g=\sum_{I:|I| \leq d} b_{I} T^{I} \in$ $K\left[T_{1}, \ldots, T_{n}\right]$ of same degree as $f$ such that $\left|a_{I}-b_{I}\right|<s^{-1} \cdot \operatorname{deg}(f)^{-1} \cdot c$. Then for all $|\cdot|^{\text {sgn }} \in\left(\mathbb{A}_{\hat{K}}^{n}\right)_{r}^{\text {an }}$ we have $|f-g|<c$. Then $|g|^{\text {sgn }}>c$ implies $|f|^{\text {sgn }}>c$ and we have shown that $\left\{|\cdot|^{\operatorname{sgn}}:|f|^{\operatorname{sgn}}>c\right\}$ contains the set $\left\{|\cdot|^{\operatorname{sgn}}:|g|^{\operatorname{sgn}}>c\right\}$ that contains $|\cdot|_{y}^{\operatorname{sgn}}$. Since the latter set is open in the topology of $\left(\mathbb{A}_{K}^{n}\right)_{r}^{\text {an }}$, this shows that the two topologies agree.

Proposition 3.6. Let $X$ be a variety over $K$ (in particular separated). Then $X_{r}^{\text {an }}$ is a Hausdorff space.

Proof. We may assume that $K$ is complete by Proposition 3.5. Let $x \neq y$ be two points in $X_{r}^{\text {an }}$. It is sufficient to construct a continuous map from an open subset of $X_{r}^{\text {an }}$ that contains $x$ and $y$ to a Hausdorff space that separates $x$ and $y$.

If there exists an open affine subscheme of $U=\operatorname{Spec}(A)$ of $X$ that contains both $p_{x}$ and $p_{y}$, we are reduced to the affine case, since $U_{r}^{\text {an }}$ is an open subset of $X_{r}^{\text {an }}$. Since $x \neq y$, there exists $f \in A$ such that $|f|_{x}^{\operatorname{sgn}} \neq|f|_{y}^{\operatorname{sgn}}$ and the map $\mathcal{M}_{r}(A) \rightarrow \mathbb{R},|\cdot|^{\operatorname{sgn}} \mapsto|f|^{\text {sgn }}$ separates $x$ and $y$.

If there does not exist such an open affine $U$, we use the canonical map $\psi: X_{r}^{\text {an }} \rightarrow X^{\text {an }}$. Since $p_{x} \neq p_{y}$, certainly $\psi(x) \neq \psi(y)$. Since $X$ is separated, $X^{\text {an }}$ is Hausdorff by Ber90, Theorem 3.4.8] and the proposition follows.

3.1. Tropicalization of $X_{r}^{\text {an }}$. Let $X$ be an affine $K$-variety. Given a family of regular functions $\mathcal{F}=\left(f_{1}, \ldots, f_{n}\right)$ on $X$, we have a natural real tropicalization map

$$
\operatorname{trop}_{r, \mathcal{F}}: X_{r}^{\mathrm{an}} \rightarrow \mathbb{R}^{n}, \quad x \mapsto\left(\left|f_{i}\right|_{x}^{\mathrm{sgn}}\right)_{i=1, \ldots, n}
$$

By construction, this map is continuous. 
Lemma 3.7. If $f_{1}, \ldots, f_{n}$ generate the coordinate ring $A$ of $X$, the map $\operatorname{trop}_{r, \mathcal{F}}$ is a proper map of topological spaces.

Proof. Let $B$ be a compact subset of $\mathbb{R}^{n}$. Since $B$ is bounded, writing $B^{\prime}=\left(\operatorname{trop}_{r, \mathcal{F}}\right)^{-1}(B)$, each $f_{i}$ is bounded on $B^{\prime}$. Since the $f_{i}$ 's generate $A$, by the ultrametric triangle inequality $\left\{|f|_{x}: x \in B^{\prime}\right\}$ is bounded for each $f \in A$. By construction, the map

$$
X_{r}^{\mathrm{an}} \rightarrow \prod_{f \in A} \mathbb{R}, \quad x \mapsto\left(|f|_{x}^{\mathrm{sgn}}\right)_{f \in A}
$$

is a homeomorphic embedding. Its image is closed, since it is defined by closed conditions $3.3(1),(2)$ and (3). Thus $B^{\prime}$ is a closed subset of $\prod_{f \in A} \mathbb{R}$.

Since all $f \in A$ are bounded on $B^{\prime}$, the image of $B^{\prime}$ is contained in a product of bounded closed intervals, thus contained in a compact subset by Tychonoff's theorem. Since $B^{\prime}$ is closed, it is compact.

Again for a family $\mathcal{F}=\left(f_{1}, \ldots, f_{n}\right)$ of elements of $A$, there is a tropicalization map on $X^{\text {an }}$ given by

$$
\operatorname{trop}_{\mathcal{F}}: X^{\text {an }} \rightarrow \mathbb{R}^{n}, \quad|\cdot|_{x} \mapsto\left(\left|f_{i}\right|_{x}\right)_{i=1, \ldots, n} .
$$

Lemma 3.8. The following map is proper:

$$
\operatorname{trop}_{r, \mathcal{F}}\left(X_{r}^{\mathrm{an}}\right) \rightarrow \operatorname{trop}_{\mathcal{F}}\left(X^{\mathrm{an}}\right), \quad\left(a_{1}, \ldots, a_{n}\right) \mapsto\left(\left|a_{1}\right|, \ldots,\left|a_{n}\right|\right) .
$$

Proof. The left hand set is a closed subset of $\mathbb{R}^{n}$ by Lemma 3.7. The map $\mathbb{R}^{n} \rightarrow \mathbb{R}_{\geq 0}^{n}$, $a \mapsto|a|$ is proper, thus so is its restriction to the closed subset trop ${ }_{r, \mathcal{F}}\left(X_{r}^{\text {an }}\right)$ of $\mathbb{R}^{n}$.

3.2. Relation with the Berkovich Analytification. Let $X$ be a $K$-variety. In this section we study $X_{r}^{\text {an }}$ via the canonical map

$$
\varphi: X_{r}^{\text {an }} \rightarrow X^{\text {an }}, \quad\left(p_{x},|\cdot|_{x}, \leq_{x}\right) \mapsto\left(p_{x},|\cdot|_{x}\right) .
$$

Lemma 3.9. The map $\varphi$ is a proper map of topological spaces.

Proof. We may assume that $X=\operatorname{Spec}(A)$ is affine. Let $\mathcal{F}=\left(f_{1}, \ldots, f_{n}\right)$ be a family of elements of $A$ that generate $A$. Let us consider the diagram

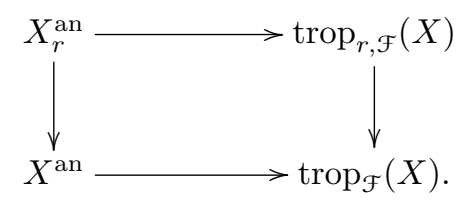

Both of the horizontal maps and the right vertical map are proper by Lemma 3.7 and 3.8 , so chasing a compact subset of $X^{\text {an }}$ through the diagram shows that the left vertical map is also proper.

Corollary 3.10. If $X$ is a proper $K$-variety, then $X_{r}^{\text {an }}$ is compact.

Proof. We may assume that $K$ is complete by Proposition 3.5. Then this follows from Lemma 3.9 since $X^{\text {an }}$ is compact by [Ber90, Theorem 3.4.8]. 
Proposition 3.11. Let $x=\left(p_{x},|\cdot|_{x}\right) \in X^{\text {an }}$, let $\Gamma_{x}$ be the value group and $k(x)$ the residue field of $|\cdot|_{x}$ on $K\left(p_{x}\right)$. Then $\varphi^{-1}(x)$ is homeomorphic to $D \times \operatorname{Sper} k(x)$, where $D$ is a discrete set of cardinality $\left|\Gamma_{x} / 2 \Gamma_{x}\right|$.

Proof. This is a direct consequence of the Baer-Krull Theorem (Theorem 2.1).

Example 3.12 (The affine line). We will now describe the affine line over $K$, where $K$ is real closed and complete with respect to a non-archimedean absolute value. We will describe $\mathbb{A}_{r}^{1 \text {,an }}$ by considering the map $\mathbb{A}_{r}^{1 \text {,an }} \rightarrow \mathbb{A}^{1 \text {,an }}$ and looking at the fibers.

We view $\mathbb{A}^{1 \text {,an }}$ as the set of pairs $x=\left(p_{x},|\cdot|_{x}\right)$ where $p_{x}$ is a point in the scheme $\mathbb{A}^{1}$ and $|\cdot|_{x}$ is an absolute value on the residue field $K\left(p_{x}\right)$. Let $k(x)$ be the residue field of the valuation ring of $K\left(p_{x}\right)$ with respect to $|.|_{x}$. We denote by $\mathcal{H}(x)$ the completion of $K\left(p_{x}\right)$ with respect to $|\cdot|_{x}$. Its residue field with respect to $|\cdot|_{x}$ is also $k(x)$. Let $K^{\prime}=K(\sqrt{-1})$ be the algebraic closure of $K$. The points in $\mathbb{A}^{1 \text {, an }}$ are classified by Berkovich into four types as follows [Ber90, p. 17].

Type I: $p_{x}$ is a closed point of $\mathbb{A}^{1}$, and $|f|_{x}=|f(a)|_{K^{\prime}}$ for some $a \in K^{\prime}$. If $a \in K$, then $p_{x}=\langle T-a\rangle$ and $K\left(p_{x}\right)=K$. Otherwise $p_{x}=\langle(T-a)(T-\bar{a})\rangle$, and $K\left(p_{x}\right)=K^{\prime}$.

Type II: $p_{x}$ is the generic point on $\mathbb{A}^{1}$, so $K\left(p_{x}\right)=K(T)$, the field of rational functions in one variable $T$ over $K$. The field $k(x)$ is of transcendence degree 1 over $k_{K}$ and $|\mathcal{H}(x)|_{x}=|K|_{K}$.

Type III: $p_{x}$ is the generic point on $\mathbb{A}^{1}, k(x)$ is algebraic over $k_{K}$ and $|\mathcal{H}(x)|_{x} /|K|_{K}$ is a free abelian group of rank 1.

Type IV: $p_{x}$ is the generic point on $\mathbb{A}^{1}, k(x)$ is algebraic over $k_{K}$ and $|\mathcal{H}(x)|_{x}=$ $|K|_{K}$.

We say that a point $x=\left(p_{x},|\cdot| x\right)$ in $\mathbb{A}^{1 \text {,an }}$ is real if there is an ordering of $K\left(p_{x}\right)$

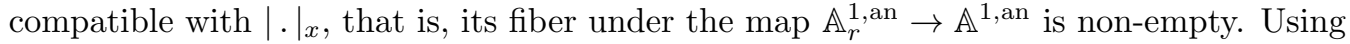
Proposition 3.11 we can describe the fibers of $x \in \mathbb{A}^{1 \text {, an }}$ under the map $\mathbb{A}_{r}^{1 \text {, an }} \rightarrow \mathbb{A}^{1 \text {, an }}$ as follows.

\begin{tabular}{l|c|c|c} 
Type & $k(x)$ & $\left|\Gamma_{x} / 2 \Gamma_{x}\right|$ & fiber \\
\hline \hline Type I, real & $k_{K}$ & 1 & one point, since $k_{K}$ has a uniqe ordering \\
\hline Type I, non-real & $k_{K^{\prime}}$ & 1 & $\varnothing$ \\
\hline Type II, real & $k_{K}(T)$ & 1 & homeomorphic to Sper $k_{K}(T)$ \\
\hline Type II, non-real & $k_{K^{\prime}}(T)$ & 1 & $\varnothing$ \\
\hline Type III, real & $k_{K}$ & 2 & two points with discrete topology \\
\hline Type III, non-real & $k_{K^{\prime}}$ & 2 & $\varnothing$ \\
\hline Type IV, real & $k_{K}$ & 1 & one point \\
\hline Type IV, non-real & $k_{K^{\prime}}$ & 1 & $\varnothing$ \\
\hline
\end{tabular}

When $k_{K}=\mathbb{R}$, such as when $K=\mathbb{R}\{\{t\}\}$, the real spectrum Sper $k_{K}(T)$ is described explicitly in Section 2. In general it can be described using Dedekind cuts.

For a point $x=\left(p_{x},\left.|\cdot|\right|_{x}\right)$ of type II or III, there exists a unique closed disc $D(a, r)=$ $\{z \in K:|z-a| \leq r\}$ where $a$ is in $K^{\prime}$ and $r$ is a positive real number, such that $|\cdot|_{x}=\sup _{c \in D(a, r)}|f(c)|_{K^{\prime}}$. The point $x$ is of type II if $r \in|K|_{K}$ and type III otherwise. It is real if and only if the disk $D(a, r)$ contains a point in $K$, which means $|\operatorname{Im}(a)| \leq r$, where $\operatorname{Im}(a)$ denotes the imaginary part of $a$. Type IV points can be constructed from 
nested sequences of discs, and the real ones are obtained as sequences of discs with real points.

3.3. Relation with the Real Spectrum. In this section, we assume that $K$ is real closed and that $|\cdot|_{K}$ is non-trivial. Then $|K|_{K}$ is necessarily dense in $\mathbb{R}_{\geq 0}$. Further let $X$ be a variety over $K$.

We first prove the following crucial lemma, which says that the absolute value of an extension field $L \supseteq K$ is determined by the position of its elements relative to $K$.

Lemma 3.13. Let $L / K$ be an extension of ordered fields with a compatible non-archimedean absolute values. Let $f \in L$ and

$$
R(f)=\left\{r \in K_{\geq 0}: r \leq \operatorname{sgn}(f) \cdot f\right\}
$$

Then $|f|=\sup \{|r|: r \in R(f)\}$.

Proof. We may assume $f \neq 0$. Replacing $f$ by $\operatorname{sgn}(f) \cdot f>0$ we may assume $f>0$. Thus if $0 \leq r \leq \operatorname{sgn}(f) \cdot f$, we have $|r| \leq|f|$, by the condition of compatibility between order and absolute value. Conversely if $|r|>|f|$, then $r>f$. Now the result follows since $|K|$ is dense in $\mathbb{R}_{\geq 0}$.

Definition 3.14. Let $X_{r}^{\max }$ be the set of closed points of $X_{r}$, and let $X_{r}^{\text {arch }} \subseteq X_{r}$ consist of those points $(p, P) \in X_{r}$ whose ordered residue field is relatively archimedean over $K$. By this we mean that, for every $b \in K(p)$, there exists $a \in K$ with $a-b \in P$.

Lemma 3.15. We have $X_{r}^{\operatorname{arch}} \subseteq X_{r}^{\max }$.

Proof. This follows from the description of specializations in the real spectrum, see KS89, III.7, in particular Kor. 5].

Proposition 3.16. The image of the canonical map $\psi: X_{r}^{\mathrm{an}} \rightarrow X_{r}$ is $X_{r}^{\mathrm{arch}}$.

Proof. Let $O_{K}=\{a \in K:|a| \leq 1\}$ be the given valuation ring of $K$. We may assume that $X=\operatorname{Spec}(A)$ is affine, so $A$ is a finitely generated $K$-algebra. The image of $X_{r}^{\text {an }}$ in $X_{r}=\operatorname{Sper}(A)$ consists of all $(p, P) \in X_{r}$ for which there is a maximal proper convex subring $B$ of $K(p)$ with $K \cap B=O_{K}$. (A subring $B$ of $K(p)$ is called convex if for any $b \in B$ and $a \in K(p)$ with $|a|<|b|$, we have $a \in B$.) Given $(p, P) \in X_{r}$, note that there always is a largest proper convex subring $B$ of $K(p)$ (since $K(p) / K$ has finite transcendence degree), and that $O_{K} \subseteq B$. By the previous remark, $(p, P) \in X_{r}^{\text {an }}$ if and only if $K \cap B=O_{K}$. This in turn holds if and only if $K$ is not contained in $B$, or equivalently, if and only if $K(p) / K$ is relatively archimedean.

Theorem 3.17. The canonical map $\psi: X_{r}^{\text {an }} \rightarrow X_{r}^{\text {arch }}$ is a homeomorphism.

Proof. This map is injective by Lemma 3.13 and surjective by Proposition 3.16

Since proving the statement for a variety $X$ clearly implies the statement for all open subvarieties, by Nagata's compactification theorem (see [Voj07, Theorem 4.1]) we may assume that $X$ is proper. Then $X_{r}^{\text {an }}$ is compact by Corollary 3.10 and $X_{r}^{\text {arch }}$ is Hausdorff.

We showed that $\psi$ is a continuous bijection from a compact space to a Hausdorff space, thus necessarily a homeomorphism. 
For the rest of this section, we view $X_{r}^{\text {an }}$ as a topological subspace of $X_{r}$ by means of the topological embedding $\psi$. Every $K$-valued point in $X(K)$ defines a point in $X_{r}^{\text {an }}$ in a canonical way.

It is well known that $X(K)$ is dense in $X_{r}$, so we have the following corollary.

Corollary 3.18. We have the following inclusions of dense subspaces of $X_{r}$ :

$$
X(K) \subseteq X_{r}^{\text {an }}=X_{r}^{\text {arch }} \subseteq X_{r}^{\max } \subseteq X_{r} .
$$

If $X$ is proper then $X_{r}^{\text {an }}$ is compact (Corollary 3.10), so we have the following, since $X_{r}^{\max }$ is Hausdorff.

Corollary 3.19. If $X$ is proper, then $X_{r}^{\max }=X_{r}^{\mathrm{an}}$.

3.4. Paths. In this section we again assume that the absolute value on $K$ is non-trivial.

While $X^{\text {an }}$ is path connected if $X$ is connected [Ber90, Theorem 3.2.1], there is no corresponding property of $X_{r}^{\text {an }}$. Indeed, we do not have any non-constant paths at all.

Theorem 3.20. Let $F:[0,1] \rightarrow X_{r}^{\text {an }}$ be a continuous map. Then $F$ is constant.

We first prove the following lemma:

Lemma 3.21. Let $X=\operatorname{Spec}(A)$ be an affine scheme, let $x \in X_{r}^{\mathrm{an}}$, and let $L$ be the real closure of the ordered quotient field of $A / p_{x}$. Denoting by $\pi:\left(\mathbb{A}^{1} \times X\right)_{r}^{\mathrm{an}} \rightarrow X_{r}^{\mathrm{an}}$ the projection, the canonical map

$$
\left(\mathbb{A}_{L}^{1}\right)_{r}^{\text {an }} \rightarrow \pi^{-1}(x) \subseteq\left(\mathbb{A}^{1} \times X\right)_{r}^{\text {an }}
$$

is a homeomorphism.

Proof. Let us denote by $\pi_{r}:\left(\mathbb{A}^{1} \times X\right)_{r} \rightarrow X_{r}$ the canonical map on real spectra. Identifying $X_{r}^{\text {an }}$ with the subset $X_{r}^{\text {arch }}$ of $X_{r}$, we have to show that $\mathbb{A}_{L, r}^{1, \text { arch }} \rightarrow \pi_{r}^{-1}(x) \cap\left(\mathbb{A}^{1} \times X\right)_{r}^{\text {arch }}$ is a homeomorphism. Since $\mathbb{A}_{L, r}^{1} \rightarrow \pi_{r}^{-1}(x)$ is a homeomorphism by [CR82, Prop. 4.3] we have to show that an order on $A[T]$ that extend the order $P_{x}$ is relatively archimedean over $K$ if and only if its extension to $L[T]$ is relatively archimedean over $L$. This is implied by the fact that $L$ is relatively archimedean over $K$, which holds since $x \in X_{r}^{\mathrm{arch}}$.

Proof of Theorem 3.20. We treat the case $X=\mathbb{A}^{1}$ first. We may assume that $K$ is complete by Lemma 3.5. Assume that $F$ is not constant. By considering the composition of $F$ with the canonical map $\varphi: X_{r}^{\text {an }} \rightarrow X^{\text {an }}$, after possibly shrinking $F$ we may assume that $F([0,1]) \cap X(K)=\varnothing$. Pick two points $|\cdot|_{1}^{\text {sgn }} \neq|\cdot|_{2}^{\text {sgn }} \in F([0,1])$. Since these define different points in $\mathbb{A}_{r}^{1}$, there exists $f \in K[T]$ such that $\operatorname{sgn}_{1}(f) \neq \operatorname{sgn}_{2}(f)$. Then $F^{-1}(V(f)) \cup F^{-1}(V(-f))$ is a cover of $[0,1]$ by disjoint non-empty open subsets, a contradiction. Note here that since the image of $F$ does not contain any rational points, $f$ does not vanish on the image of $F$.

Now assume the statement is known for $\mathbb{A}^{n}$. Let $F:[0,1] \rightarrow \mathbb{A}_{r}^{n+1, \text { an }}$. If we denote by $\pi: \mathbb{A}_{r}^{n+1, \text { an }} \rightarrow \mathbb{A}_{r}^{n}$, an the projection, then $\pi \circ F$ has to be constant by the inductive hypothesis, thus $F([0,1])$ is contained in a fiber of $\pi^{-1}(x)$. By Lemma 3.21 this fiber is homeomorphic to $\mathbb{A}_{L, r}^{1, \text { an }}$ for an extension $L / K$. Again by the inductive hypothesis, $F$ has to be constant.

The case of general $X$ follows from the affine case, which in turn follows from the $\mathbb{A}^{n}$ case. 


\section{Semialgebraic Sets}

As before, let $K$ be a real closed field with a non-archimedean absolute value, and $X=\operatorname{Spec}(A)$ an affine $K$-variety. Let $S \subseteq X(K)$ be a semialgebraic subset.

We denote by $\tilde{S}$ the constructible subset of $X_{r}=$ Sper $A$ associated with the semialgebraic set $S$. In particular, if $S=\bigcup_{i=1}^{m}\left\{\xi \in X(K): \operatorname{sgn} f_{i j}(\xi)=\epsilon_{i j} j=1, \ldots, r\right\}$ is any finite semialgebraic description of $S$ (with $f_{i j} \in A$ and $\epsilon_{i j} \in\{-1,0,1\}$ ), then $\tilde{S}$ has the same description in the real spectrum, i.e.

$$
\tilde{S}=\bigcup_{i=1}^{m}\left\{x \in \operatorname{Sper} A: \operatorname{sgn}_{x}\left(f_{i j}\right)=\epsilon_{i j}, i=1, \ldots, r\right\},
$$

and the mapping $S \mapsto \tilde{S}$ is compatible with boolean combinations and taking closure and interior. This construction does not depend on the choice of the semialgebraic description of $S$ [BCR98, Proposition 7.2.2].

Recall the we denote by $\psi: X_{r}^{\text {an }} \rightarrow X_{r}$ the canonical map.

Definition 4.1. The real analytification $S_{r}^{\text {an }}$ of $S$ is the preimage of $\tilde{S}$ under $\psi$ :

$$
S_{r}^{\text {an }}:=\psi^{-1}(\tilde{S}) \text {. }
$$

In particular, if $S=X(K)$, this definition gives $S_{r}^{\text {an }}=X_{r}^{\text {an }}$. Note that $S_{r}^{\text {an }}$ is a Hausdorff topological space. It follows directly from the definitions that the construction $S \mapsto S_{r}^{\text {an }}$ is compatible with boolean combinations.

Assume for the rest of this section that the absolute value on $K$ is non-trivial. Then $\psi$ is a homeomorphism onto its image by Theorem 3.17 and forming $S_{r}^{\text {an }}$ is compatible with taking closures and interiors.

We generalize Corollaries 3.18 and 3.19 to the semialgebraic case, and we prove a converse to the latter:

Proposition 4.2. Let $S \subseteq X(K)$ be a semialgebraic set as above.

(a) The inclusions $S \subseteq S_{r}^{\text {an }}=\tilde{S}^{\text {arch }} \subseteq \tilde{S}^{\text {max }}$ of Hausdorff spaces hold, and $S$ is dense in $\tilde{S}^{\max }$.

(b) Equality $S_{r}^{\text {an }}=\tilde{S}^{\text {max }}$ holds if and only if $S$ is semialgebraically compact, and if and only if $S_{r}^{\text {an }}$ is compact.

Proof. (a) The inclusions are clear, and $S$ is well known to be dense in $\tilde{S}$. For (b) we have to show: $\tilde{S}^{\text {max }} \subseteq \tilde{S}^{\text {arch }} \Leftrightarrow S$ is semialgebraically (s.a.) compact. This is well-known, but for lack of a suitable reference we indicate a proof: If $S$ is s.a. compact, then for every $f \in K[X]$ there is $c \in K$ with $f<c$ on $S$. So from [KS89, III.7] we see that every point in $\tilde{S}$ specializes to a point in $\tilde{S}^{\text {arch }}$. Conversely, if $S$ is not s.a. compact, there exists a semialgebraic curve in $S$ without an endpoint in $S$. Associated with it is a point in $\tilde{S}^{\max } \backslash \widetilde{S}^{\text {arch }}$.

It is easy to see that the spaces $S_{r}^{\text {an }}$ are functorial with respect to morphisms of $K$ varieties. In fact they are functorial also with respect to semialgebraic maps. Recall that a map $\phi: S \rightarrow N$ (between semialgebraic subsets of affine $K$-varieties) is called semialgebraic if $\phi$ is continuous and its graph is a semialgebraic subset of $S \times N$. 
Lemma 4.3. Let $\phi: S \rightarrow N$ be a semialgebraic map. There is a unique continuous map $\phi_{*}: S_{r}^{\text {an }} \rightarrow N_{r}^{\text {an }}$ making the diagram

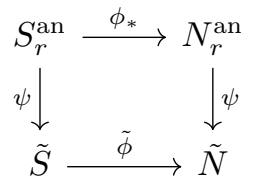

commute, where $\tilde{\phi}$ is the map induced by $\phi$ in the real spectrum.

Proof. The map $\tilde{\phi}$ satisfies $\tilde{\phi}\left(\tilde{S}^{\text {arch }}\right) \subseteq \tilde{N}^{\text {arch }}$, since for every $x \in \tilde{S}, \phi$ induces an embedding $K(\tilde{\phi}(x)) \rightarrow K(x)$ of the ordered residue fields. Therefore the restriction $\tilde{S}^{\text {arch }} \rightarrow \tilde{N}^{\text {arch }}$ of $\tilde{\phi}$ has the desired properties, upon making the identifications $S_{r}^{\text {an }}=\tilde{S}^{\text {arch }}$ and $N_{r}^{\text {an }}=\tilde{N}^{\text {arch }}$. Uniqueness is clear since $N_{r}^{\text {an }}$ is Hausdorff and $S$ is dense in $S_{r}^{\text {an }}$.

Theorem 4.4. Let $X$ be an affine $K$-variety, let $S \subseteq X(K)$ be a semialgebraic set, with corresponding constructible subset $\tilde{S}$ of $X_{r}$. The map $\psi: X_{r}^{\text {an }} \rightarrow X_{r}$ induces a bijection between the connected components of $\tilde{S}$ and those of $S_{r}^{\text {an }}$ : For any connected component $C$ of $\tilde{S}$, the preimage of $C$ in $S_{r}^{\text {an }}$ is connected.

Note that in turn, the connected components of $\tilde{S}$ are in natural bijection with the semialgebraic connected components of $S$.

Proof. It suffices to show that when $S$ is semialgebraically connected, then $S_{r}^{\text {an }} \cong S^{\text {arch }}$ is connected as well. Let $U_{i} \subseteq X_{r}(i=1,2)$ be open subsets such that $\tilde{S}^{\text {arch }} \subseteq U_{1} \cup U_{2}$ and $\tilde{S}^{\text {arch }} \cap U_{1} \cap U_{2}=\varnothing$, and assume $\tilde{S}^{\text {arch }} \cap U_{i} \neq \varnothing$ for $i=1,2$. Then for $i=1,2$ there exists a $K$-rational point $P_{i}$ in $S \cap U_{i}$. Let $J=[0,1]_{K}$. Since $S$ is semialgebraically pathconnected, there exists a semialgebraic path $\gamma: J \rightarrow S$ from $P_{1}$ to $P_{2}$. Using Lemma4.3, it follows that $J_{r}^{\text {an }}$ is disconnected. But $J$ is semialgebraically compact, and so $J_{r}^{\text {an }}=J_{r}^{\max }$ is connected as well, a contradiction.

\section{Hyperfields and Tropicalization of Inequalities}

5.1. Hyperfields. A hyperfield/hyperring $\mathbb{H}$ is a set with a multiplication · and addition $\oplus$, where addition may be multivalued, that satisfies a set of axioms similar to those for a field/ring. We first recall some useful hyperfields as introduced by Viro in Vir10, where definitions can be found.

- The hyperfield of signs $\mathbb{S}$ has multiplicative group $(\{ \pm 1\}, \cdot)$ with addition where 0 is the neutral element and $1 \oplus 1=1,-1 \oplus-1=-1$ and $-1 \oplus 1=\mathbb{S}$.

- The tropical hyperfield $\mathbb{T}$, with multiplicative notation, has as multiplicative group $\left(\mathbb{R}_{>0}, \cdot\right)$, and the addition is defined by

$$
a \oplus b=\left\{\begin{array}{l}
\max (a, b) \text { if } a \neq b \\
{[0, a] \text { if } a=b .}
\end{array}\right.
$$


- The real tropical hyperfield $\mathbb{R} \mathbb{T}$ has multiplicative group $\left(\mathbb{R}^{*}, \cdot\right)$ with addition

$$
a \oplus b=\left\{\begin{array}{l}
a \text { if }|a|>|b| \\
b \text { if }|a|<|b| \\
a \text { if } a=b \\
{[a, b] \text { if } a=-b \leq 0} \\
{[b, a] \text { if } a=-b \geq 0 .}
\end{array}\right.
$$

A morphism of hyperfields $\varphi: \mathbb{H}_{1} \rightarrow \mathbb{H}_{2}$ is a map which induces a morphism of multiplicative groups and satisfies $\varphi(x \oplus y) \subseteq \varphi(x) \oplus \varphi(y)$. It is a direct consequence of the definitions that a seminorm on a ring $A$ is the same as a morphism of hyperrings $A \rightarrow \mathbb{T}$. Similarly, a signed seminorm on $A$ is an morphism $A \rightarrow \mathbb{R T}$ and an ordering on $A$ is a morphism $A \rightarrow \mathbb{S}$.

Let $\mathbb{H}$ be a hyperfield with a topology and let $K$ be a field with a morphism $K \rightarrow \mathbb{H}$. Let $A$ be a $K$-algebra. Denote by $\operatorname{Hom}_{K}(A, \mathbb{H})$ the set of homomorphisms of hyperrings $\varphi: A \rightarrow \mathbb{H}$ which extend the given morphism on $K$. We endow it with the coarsest topology such that for all $f \in A$ the map $\operatorname{Hom}_{K}(A, \mathbb{H}) \rightarrow \mathbb{H}, \varphi \mapsto \varphi(f)$ is continuous.

Jun showed that we have canonical homeomorphisms

$$
\operatorname{Hom}_{K}(A, \mathbb{T})=\mathcal{M}(A) \text { and } \operatorname{Hom}_{K}(A, \mathbb{S})=\operatorname{Sper}(A),
$$

when $\mathbb{T}$ has the topology of $\mathbb{R}_{\geq 0}$ and $\mathbb{S}$ has the topology of $\mathbb{R} / \mathbb{R}_{>0}$ [Jun17, Proposition 5.8 \& Lemma 6.11].

From this point of view, one can furthermore canonically see the tropicalization map. Given a family $\mathcal{F}=\left(f_{1}, \ldots, f_{n}\right)$ we have

$$
\begin{aligned}
\operatorname{trop}_{\mathcal{F}}: \operatorname{Hom}_{K}(A, \mathbb{T}) & \rightarrow \mathbb{T}^{n}, \\
\varphi & \mapsto\left(\varphi\left(f_{1}\right), \ldots, \varphi\left(f_{n}\right)\right) .
\end{aligned}
$$

Our definition of $X_{r}^{\text {an }}$ is inspired by Jun's work, and we have

$$
\begin{aligned}
\mathcal{M}_{r}(A)=\operatorname{Hom}_{K}(A, \mathbb{R} \mathbb{T}) \quad \text { and } \operatorname{trop}_{r, \mathcal{F}}: \operatorname{Hom}_{K}(A, \mathbb{R} \mathbb{T}) & \rightarrow \mathbb{R} \mathbb{T}^{n}, \\
\varphi & \mapsto\left(\varphi\left(f_{1}\right), \ldots, \varphi\left(f_{n}\right)\right) .
\end{aligned}
$$

Jun also globalizes these constructions and defines the set of $\mathbb{H}$-rational points $X_{K}(\mathbb{H})$ for not necessarily affine $K$-varieties $X$. He also shows the analogous results

$$
X^{\text {an }}=X_{K}(\mathbb{T}) \text { and } X_{r}=X_{K}(\mathbb{S}) .
$$

Similarly one can show

$$
X_{r}^{\mathrm{an}}=X_{K}(\mathbb{R} \mathbb{T})
$$

5.2. Real Tropicalization of $K^{n}$ and Tropicalizing Polynomial Inequalities. For a hyperfield $\mathbb{H}$, a polynomial in the variables $x_{1}, \ldots, x_{n}$ over $\mathbb{H}$ is a formal expression $F=\bigoplus_{d_{1}, \ldots, d_{n} \in \mathbb{Z}} a_{d_{1}, \ldots, d_{n}} x_{1}^{d_{1}} \cdots x_{n}^{d_{n}}$ where all but finitely many coefficients $a_{d_{1}, \ldots, d_{n}}$ are zero. For $c=\left(c_{1}, \ldots, c_{n}\right) \in \mathbb{H}^{n}$ we have $F(c)=\bigoplus_{d_{1}, \ldots, d_{n}} a_{d_{i}, \ldots, d_{n}} c_{1}^{d_{i}} \cdots c_{n}^{d_{n}} \subseteq \mathbb{H}$.

Given a tropical polynomial $F$ in $n$ variables over the real tropical hyperfield $\mathbb{R} \mathbb{T}$, we define the tropical semialgebraic sets in $\mathbb{R T}^{n}$ defined by $F=0, F \geq 0$, and $F>0$ to be, 
respectively, the sets

$$
\begin{aligned}
& \{F=0\}:=\left\{\left(x_{1}, \ldots, x_{n}\right) \in \mathbb{R}^{n}: F\left(x_{1}, \ldots, x_{n}\right) \ni 0\right\} \\
& \{F \geq 0\}:=\left\{\left(x_{1}, \ldots, x_{n}\right) \in \mathbb{R T}^{n}: F\left(x_{1}, \ldots, x_{n}\right) \text { contains a nonnegative number }\right\} \\
& \{F>0\}:=\left\{\left(x_{1}, \ldots, x_{n}\right) \in \mathbb{R}^{n}: F\left(x_{1}, \ldots, x_{n}\right) \text { is a positive number }\right\} .
\end{aligned}
$$

Recall that the tropical addition may be multivalued, and $F\left(x_{1}, \ldots, x_{n}\right)$ is either a point or an interval of the form $[-a, a]$.

For a polynomial $f=\sum a_{d_{1}, \ldots, d_{n}} x_{1}^{d_{1}} \ldots x_{n}^{d_{n}} \in K\left[x_{1}, \ldots, x_{n}\right]$, let $\operatorname{trop}_{r}(f)$ be the polynomial $\bigoplus\left|a_{d_{1}, \ldots, d_{n}}\right|^{\operatorname{sgn}} x_{1}^{d_{1}} \ldots x_{n}^{d_{n}}$ over $\mathbb{R} \mathbb{T}$ obtained by replacing + with $\oplus$ and each coefficient with its signed seminorm. For any $a \in K^{n}$, we have $|f(a)|^{\text {sgn }} \in \operatorname{trop}_{r}(f)\left(|a|^{\operatorname{sgn}}\right)$ because the signed seminorm is a morphism of hyperfields from $K$ to $\mathbb{R} \mathbb{T}$. In particular, if a point $a \in K^{n}$ satisfies $f \geq 0$, then $|a|^{\text {sgn }}$ satisfies $\operatorname{trop}_{r}(f)\left(|a|^{\text {sgn }}\right) \geq 0$. On the other hand, if a point $a \in K^{n}$ satisfies $\operatorname{trop}_{r}(f)\left(|a|^{\text {sgn }}\right)>0$, then $f(a)>0$.

In general, even if $K \rightarrow \mathbb{R T}$ is surjective, it is not the case that

$$
\operatorname{trop}_{r}\left(\left\{x \in K^{n}: f(x) \geq 0\right\}\right)=\left\{\operatorname{trop}_{r}(f)(x) \geq 0\right\} .
$$

For example, let $S=\left\{(x, y) \in K^{2}:(x-2)^{2}+(y-2)^{2} \leq 1\right\}$. Then $\operatorname{trop}_{r}(S)$ consists of a single point $(1,1)$. However

$$
\operatorname{trop}_{r}\left((x-2)^{2}+(y-2)^{2}-1\right)=X^{2} \oplus Y^{2} \oplus-X \oplus-Y \oplus 1 \leq 0
$$

cuts out two line segments which join $(1,1)$ to $(-1,1)$ and $(1,-1)$ respectively. For more examples, see Figures 12, and 3. We will see in Theorem 6.9 that tropicalizing all polynomial inequalities satisfied by a semialgebraic set $S$ cuts out trop $r$, if $K \rightarrow \mathbb{T} \mathbb{R}$ is surjective.

\section{The Fundamental Theorem and Limit of Tropicalizations}

In this section we let $S \subseteq K^{n}$ be a semialgebraic set, with Zariski closure $X$ in $\mathbb{A}^{n}$. We denote by $A$ the coordinate ring of $X$.

6.1. Density. Assume that the absolute value on $K$ is non-trivial.

Theorem 6.1 (Weak Density). Let $\mathcal{F}=\left(f_{1}, \ldots, f_{n}\right)$ be a family of elements in $A$. Then $\operatorname{trop}_{r, \mathcal{F}}(S)$ is dense in $\operatorname{trop}_{r, \mathcal{F}}\left(S_{r}^{\text {an }}\right)$.

Proof. This follows since $S$ is dense in $S_{r}^{\text {an }} 4.2$ and $\operatorname{trop}_{r, \mathcal{F}}$ is a continuous map.

Theorem 6.2 (Strong Density). Let $\mathcal{F}=\left(f_{1}, \ldots, f_{n}\right)$ be a family of elements in $A$. Then

$$
\operatorname{trop}_{r, \mathcal{F}}\left(S_{r}^{\mathrm{an}}\right) \cap\left(|K|_{K}^{\mathrm{sgn}}\right)^{m}=\operatorname{trop}_{r, \mathcal{F}}(S) .
$$

This statement is true by model-theoretic reasons (Cherlin-Dickmann quantifier elimination for real closed fields with compatible valuations CD83]). We nevertheless give a proof with our methods.

Proof. The inclusion $\supseteq$ is immediate, so we need to prove the opposite inclusion. After possibly shrinking $S$, we may assume it is basic semialgebraic, i.e. defined by inequalities $g_{1} \geq 0, \ldots, g_{s} \geq 0$ and $h_{1}>0, \ldots, h_{r}>0$. 


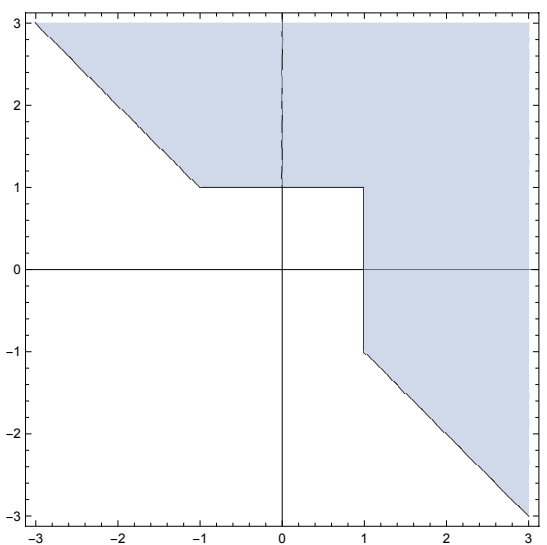

The subset of $\mathbb{R T}^{2}$ defined by

$$
X \oplus Y \oplus-1 \geq 0 .
$$

It coincides with the real tropicalization of, e.g.

$\left\{(x, y) \in K^{2}: 2 x+3 y-5 \geq 0\right\}$.

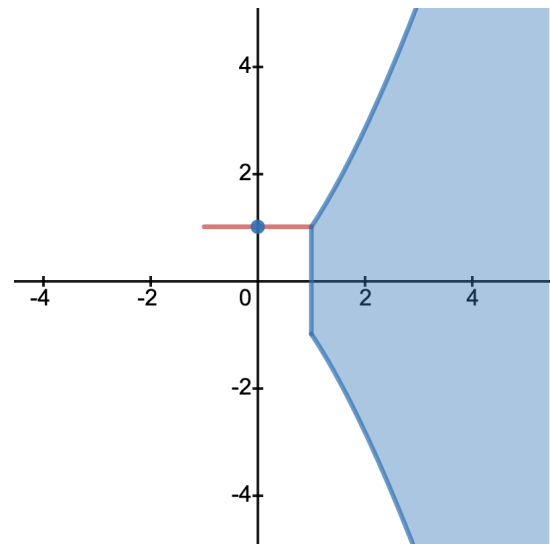

The subset of $\mathbb{R}^{2}$ defined by

$$
X^{3} \oplus Y \oplus-X^{2} \oplus-Y^{2} \oplus-1 \geq 0 .
$$

The real tropicalization of

$$
\left\{(x, y) \in K^{2}: x^{3}+2 y-x^{2}-y^{2}-1 \geq 0\right\}
$$

consists of the shaded region and the point $(0,1)$, but not the red segment. Compare with Figure 7 in Ale13.

FiguRE 1. Real tropicalizations of sets and polynomial inequalities.

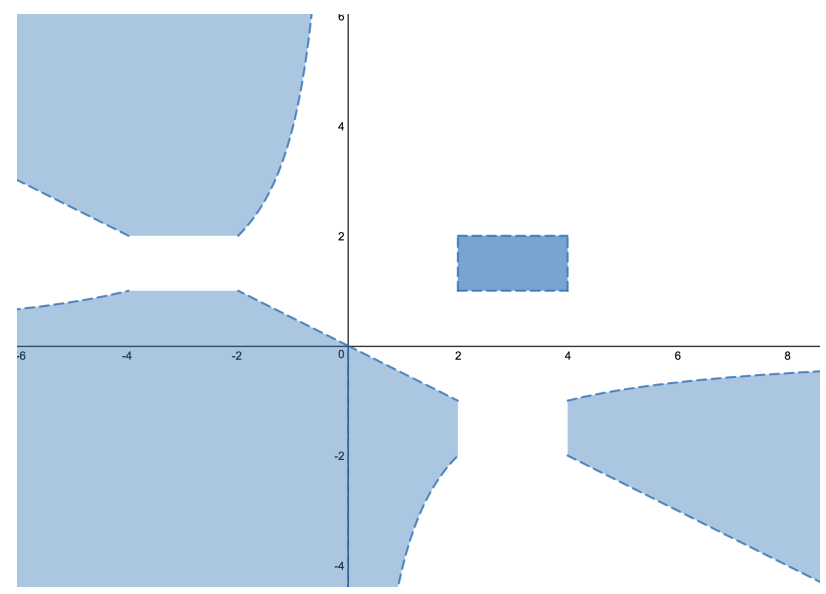

Figure 2. The open subset of $\mathbb{R}^{2}$ defined by

$$
x y\left(-1 \oplus \frac{2}{x} \oplus \frac{x}{4} \oplus \frac{1}{y} \oplus \frac{y}{2}\right)<0 .
$$


Let $z \in \operatorname{trop}_{r, \mathcal{F}}\left(S_{r}^{\mathrm{an}}\right) \cap\left(|K|_{K}^{\mathrm{sgn}}\right)^{m}$. Let $x=|\cdot|_{x}^{\mathrm{sgn}} \in S_{r}^{\text {an }} \operatorname{such}$ that $\operatorname{trop}_{\mathcal{F}}(x)=z$. After reordering we may assume $\left|g_{1}\right|_{x}=0, \ldots,\left|g_{t}\right|_{x}=0,\left|g_{t+1}\right|_{x}>0, \ldots,\left|g_{s}\right|_{x}>0$. Replacing $\mathcal{F}$ by $\left(f_{1}, \ldots, f_{m}, g_{1}, \ldots, g_{t}\right), z=\left(z_{1}, \ldots, z_{n}\right)$ by $\left(z_{1}, \ldots, z_{n},\left|g_{1}\right|_{x}^{\text {sgn }}, \ldots,\left|g_{t}\right|_{x}^{\text {sgn }}\right)$, and $h_{1}, \ldots, h_{r}$ by $h_{1}, \ldots, h_{r}, g_{t+1}, \ldots, g_{s}$ we may assume that all inequalities are strict.

We may also assume that none of the coordinates of $z$ are zero by replacing $X$ with the vanishing locus of $f_{i}$ if $z_{i}=0$ and $S$ by its intersection with this set. After possibly replacing $f_{i}$ by $c_{i} \cdot f_{i}$, where $\left|c_{i}\right|_{K}^{\text {sgn }}=z_{i}^{-1}$ we may assume that $z=(1, \ldots, 1)$.

Consider the map $\varphi: X_{r}^{\text {an }} \rightarrow X^{\text {an }}$. Then

$$
\varphi^{-1}\{\varphi(x)\}=D \times \operatorname{Sper} k_{K(x)}
$$

by the Baer-Krull theorem 2.1, where $D$ is a finite discrete set. Let $d \in D$ such that $x \in\{d\} \times \operatorname{Sper} k_{K(x)}$ and denote $E:=\{d\} \times$ Sper $k_{K(x)}$.

Let

$$
U=\left\{|\cdot|^{\operatorname{sgn}}:\left|f_{i}\right|^{\text {sgn }}>0 \text { and }\left|h_{j}\right|^{\text {sgn }}>0 \text { for all } i, j\right\} \subseteq X_{r}^{\text {an }}
$$

and $V=E \cap U$. Then $V$ is an open subset of Sper $k_{K(x)}$ that contains $x$, thus there exist $\tilde{c}_{1}, \ldots, \tilde{c}_{k} \in k_{K(x)}$ such that

$$
x \in\left\{P \in \operatorname{Sper} k_{K(x)}: \tilde{c}_{j}>_{P} 0 \text { and } \tilde{f}_{i}>_{P} 0 \text { for all } i, j\right\} \subseteq V
$$

By Lemma 6.3 below, there exists $Q \in \operatorname{Sper} k_{K(x)}$ and $\tilde{C} \in k_{K}$ such that

$$
\tilde{C}>_{Q} \tilde{C}_{j}^{ \pm 1}>_{Q} 0 \text { and } \tilde{C}>_{Q} \tilde{f}_{i}^{ \pm 1}>_{Q} 0 \text { for all } i, j .
$$

Then $>_{Q}$ defines a point in $V$ and consequently a point $|\cdot|_{Q}^{\text {sgn }}$ in $X_{r}^{\text {an }} \subseteq X_{r}$. Let $C \in K$ such that $|C|=1$ and the residue class of $C$ in $k_{K}$ is $\tilde{C}$. Then

$$
\left\{P \in X_{r}: C^{-1}<_{P} f_{i}<_{P} C \text { and } h_{j}>_{P} 0 \text { for all } i, j\right\}
$$

is an open subset of $X_{r}$ containing $|\cdot|_{Q}^{\text {sgn }}$. Thus this set is non-empty and contains a rational point $w \in X(K)$. Since

$$
C^{-1}<f_{i}(w)<C \text { we have }\left|f_{i}(w)\right|_{K}^{\text {sgn }}=1
$$

and since $h_{j}(w)>0$ meaning $w \in S$ and $\operatorname{trop}_{\mathcal{F}}(w)=z$.

We used the following lemma:

Lemma 6.3. Let $F$ be a real closed field and $E$ a finitely generated extension of $F$. Let $f_{1}, \ldots, f_{n} \in E$. Assuming that there exists an order of $E$ where all the $f_{i}$ are positive, there also exists an order $P$ of $E$ where all the $f_{i}$ are positive and bounded over $F$ (in the sense that there exists $c \in F$ such that $c>_{P} f_{i}$ for all $i$ ).

Proof. Choose an affine integral $F$-variety $V$ for which $F(V) \cong E$ and $f_{1}, \ldots, f_{n} \in F[V]$. The semialgebraic set $\left\{\xi \in V(F): f_{i}(\xi)>0, i=1, \ldots, n\right\}$ contains a nonsingular point $\xi$ of $V$. Since $\xi$ is nonsingular, there exists an ordering $P$ of $F(V)$ specializing into $\xi$ inside Sper $F[V]$. Any such $\beta$ satisfies the condition of the lemma, since $f_{i}<_{P} c$ for $c:=1+\max \left\{f_{1}(\xi), \ldots, f_{n}(\xi)\right\}$. 
6.2. Fundamental Theorem. Let $K$ be a real closed field with a compatible absolute value (which might be trivial). Let $L / K$ be an extension of real closed fields where the absolute value on $L$ extends the one on $K$ and is not trivial. Let $M / L$ be an extension of real closed fields where the absolute value on $M$ extends the one on $L$ and $|M|=\mathbb{R}_{\geq 0}$.

Let $S$ be a semialgebraic set defined over $K$ and let $S_{M}$ and $S_{L}$ be the base-changes to $M$ and $L$, respectively. Let $A$ be the coordinate ring of the Zariski closure of $S$ and $A_{L}:=A \otimes_{K} L$ the base-change of $A$ to $L$.

Let $\sigma \in\{1,-1,0\}^{n}$ and denote by $S^{\sigma}=\left\{x \in S \mid \operatorname{sgn}\left(x_{i}\right)=\sigma_{i}\right\}$ and similarly for $\mathbb{R}^{\sigma}$, $M^{\sigma}, K^{\sigma}$ and $L^{\sigma}$. We first show that the tropicalization of any semialgebraic set coincides with the tropicalization of its closure.

Lemma 6.4. For any semialgebraic set $S$ in $K^{n}$ and any $\sigma \in\{1,-1,0\}^{n}$, we have

$$
\operatorname{trop}_{r}\left(S^{\sigma}\right)=\operatorname{trop}_{r}\left(\overline{S^{\sigma}}\right) \cap \mathbb{R}^{\sigma} .
$$

Proof. The statement is immediate when the absolute value on $K$ is trivial, so we may assume it is non-trivial.

The inclusion $\subseteq$ is obvious.

Now let $y \in \operatorname{trop}\left(\overline{S^{\sigma}}\right) \cap \mathbb{R}^{\sigma}$. Let $x \in \overline{S^{\sigma}}$ such that $\operatorname{trop}_{r}(x)=y$ and let $\left(x^{i}\right)_{i \in \mathbb{N}}$ be a sequence of points in $S^{\sigma}$ that converges to $x$. Since the absolute value is non-trivial, the topology on $K^{n}$ induced by the order is the same as the one induced by the absolute value. Thus there exists $N \in \mathbb{N}$ such that $\left|x_{j}^{i}-x_{j}\right|<\left|x_{j}\right|$ for all $i \geq N$ and for all $j$ with $x_{j} \neq 0$. By the non-archimedean triangle inequality this implies $\left|x_{j}^{i}\right|=\left|x_{j}\right|$ for all $i \geq N$. In particular $y=\operatorname{trop}_{r}\left(x^{N}\right) \in \operatorname{trop}_{r}\left(S^{\sigma}\right)$.

Definition 6.5. Let $\Gamma$ be a subgroup of $\mathbb{R}$. An integral $\Gamma$-affine polyhedron in $\mathbb{R}^{n}$ is a set of the following form

$$
\left\{X \in \mathbb{R}^{n} \mid \alpha_{i} \cdot X+C_{i} \leq 0 \text { for } i=1,2, \ldots, m\right\},
$$

and an open integral $\Gamma$-affine polyhedron in $\mathbb{R}^{n}$ is a set of the form

$$
\left\{X \in \mathbb{R}^{n} \mid \alpha_{i} \cdot X+C_{i}<0 \text { for } i=1,2, \ldots, m\right\}
$$

where $\alpha_{1}, \ldots, \alpha_{m}$ are integer vectors, and $C_{1}, \ldots, C_{m}$ are in $\Gamma$.

Lemma 6.6. Let $P$ be a open $\Gamma$-affine polyhedron polyhedron in $\mathbb{R}^{n}$. Then there exists a real tropical polynomial $F_{P}$ with coefficients in $\pm \exp (\Gamma)$ such that $P=\log \left\{F_{P}>0\right\}$.

Proof. We write

$$
P=\left\{\max \left(\left(\alpha_{1} \cdot X+C_{1}\right), \ldots,\left(\alpha_{m} \cdot X+C_{m}\right)\right)>0\right\} .
$$

with $\alpha_{1}, \ldots, \alpha_{m} \in \mathbb{Z}^{n}$ and $C_{1}, \ldots, C_{m} \in \Gamma$. One immediately checks that picking

$$
F_{P}=A_{1} x^{\alpha_{1}} \oplus \cdots \oplus A_{m} x^{\alpha_{m}} \oplus(-1)
$$

where $A_{i}=\exp \left(C_{i}\right)$ suffices.

The next two lemmas are used to prove the $(3) \subseteq(4)$ part of Theorem 6.9

Lemma 6.7. Let $T$ be a finite union of integral $\Gamma$-affine polyhedra in $\mathbb{R}^{n}$. Then the complement of $T$ is a finite union of open integral $\Gamma$-affine polyhedra. 
Proof. To see that $T$ has such a description, first write $T$ as a union of cells in an arrangement of hyperplanes defined by $f_{1}=0, f_{2}=0, \ldots, f_{m}=0$ where each $f_{i}$ has the form $\alpha \cdot X+C$ with $\alpha \in \mathbb{Z}^{n}, C \in \Gamma$. This can be done, for example, by taking all the hyperplanes defining the polyhedra that make up $T$. A hyperplane arrangement partitions the ambient space into relatively open polyhedra, each defined by a collection of strict inequalities and equations of the form $f_{i}>0, f_{j}<0$, or $f_{k}=0$.

Let $\tau$ be a relatively open cell in the hyperplane arrangement which is not contained in $T$. Let $P_{\tau}$ be the open polyhedron defined by all the inequalities $f_{i}>0$ such that $f_{i}(\tau)>0$ and $f_{j}<0$ such that $f_{j}(\tau)<0$. Clearly, $\tau$ is in $P_{\tau}$. We claim that $P_{\tau} \cap T=\varnothing$. Let $\tau^{\prime}$ be a cell whose relative interior is in $P_{\tau}$. Then $\tau^{\prime}$ is defined by the inequalities defining $P_{\tau}$ and possibly some additional equations of the form $f_{k}=0$. But these additional equations must be satisfied by $\tau$ as well, so $\tau$ is in the closure of $\tau^{\prime}$. Since $T$ is closed and $\tau$ is not in $T$, it follows that $\tau^{\prime}$ is not in $T$.

Thus for every relatively open cell $\tau$ which is not in $T$, we have found an open polyhedron $P_{\tau}$ which contains $\tau$ and does not intersect $T$. Since the hyperplane arrangement consists of finitely many cells, we have found a description of $T$ as the intersection of complements of finitely many open polyhedra.

Next, we will prove a lifting lemma for inequalities.

Lemma 6.8. Let $S \subseteq K_{>0}^{n}$ be a semialgebraic set and $F$ be a tropical polynomial with coefficients in $|K|_{K}^{\mathrm{sgn}}$ such that $F \geq 0$ on $\operatorname{trop}_{r}(S)$. Then there is a polynomial $f$ in $K\left[x_{1}, \ldots, x_{n}\right]$ such that $f \geq 0$ on $S$ and $\operatorname{trop}(f)=F$.

Proof. Suppose $F \geq 0$ on $\operatorname{trop}_{r}(S)$, and $F$ has the form

$$
F\left(X_{1}, \ldots, X_{n}\right)=A_{1} X^{\alpha_{1}} \oplus \cdots \oplus A_{k} X^{\alpha_{k}} \oplus B_{1} X^{\beta_{1}} \oplus \cdots \oplus B_{\ell} X^{\beta_{\ell}}
$$

where $A_{1}, \ldots, A_{k}$ are positive elements and $B_{1}, \ldots, B_{\ell}$ are negative elements of $|K|_{K}^{\mathrm{sgn}}$. Consider a polynomial of the form

$$
f_{\varepsilon}\left(x_{1}, \ldots, x_{n}\right)=a_{1} x^{\alpha_{1}}+\cdots+a_{k} x^{\alpha_{k}}+\varepsilon\left(b_{1} x^{\beta_{1}}+\cdots+b_{\ell} x^{\beta_{\ell}}\right)
$$

where $\left|a_{i}\right|_{K}^{\mathrm{sgn}}=A_{i}$ and $\left|b_{j}\right|_{K}^{\mathrm{sgn}}=B_{j}$ for each $i=1, \ldots, k$ and $j=1, \ldots, \ell$. We wish to show that $f_{\varepsilon}>0$ on $S$ for some positive $\varepsilon \in K$ with $|\varepsilon|_{K}=1$.

Consider the map

$$
\varphi: S \rightarrow K, x \mapsto \frac{a_{1} x^{\alpha_{1}}+\cdots+a_{k} x^{\alpha_{k}}}{-\left(b_{1} x^{\beta_{1}}+\cdots+b_{\ell} x^{\beta_{\ell}}\right)} .
$$

For any $x \in S \subseteq K_{>0}^{n}$, we have $|x|_{K}^{\mathrm{sgn}} \in \operatorname{Trop}_{r}(S)$, so $F\left(|x|_{M}^{\mathrm{sgn}}\right) \geq 0$. Then we have

$$
\left|a_{1} x^{\alpha_{1}}+\cdots+a_{k} x^{\alpha_{k}}\right|_{K}^{\mathrm{sgn}} \geq\left|-\left(b_{1} x^{\beta_{1}}+\cdots+b_{\ell} x^{\beta_{\ell}}\right)\right|_{K}^{\mathrm{sgn}}>0,
$$

which implies that $|\varphi(x)|_{K}^{\mathrm{sgn}} \geq 1$. The image $\varphi(S)$ is a $K$-semialgebraic subset of $K$, so there is an element $0<\varepsilon \in K$ with $|\varepsilon|_{K}=1$ such that $\varphi(S) \geq \varepsilon$. This means $f_{\varepsilon}>0$ on $S$.

We will now give an analogue of the Fundamental Theorem of Tropical Geometry, for semialgebraic sets. Compare with [Dra08, Theorem 4.2], EKL06], and [MS15, §3.2].

Theorem 6.9. Let $S$ be a semialgebraic set in $K^{n}$ defined over $K$. The following subsets of $\mathbb{R}^{n}$ agree. 
(1) The tropicalization of the real analytification $S_{r}^{\text {an }}$ of $S$ :

$$
\operatorname{trop}_{r}\left(S_{r}^{\text {an }}\right) \text {; }
$$

(2) The tropicalization of the $M$-points of $S$ :

$$
\operatorname{trop}_{r}\left(S_{M}\right)
$$

(3) The set defined by tropicalization of weak inequalities in $K\left[T_{1}, \ldots, T_{n}\right]$ satisfied by $S$ in each orthant:

$$
\bigcup_{\sigma \in\{+,-, 0\}^{n}}\left(\bigcap_{f \geq 0 \text { on } S^{\sigma}}\{\operatorname{trop}(f) \geq 0\} \cap \mathbb{R}^{\sigma}\right) ;
$$

The intersection may be taken to be finite.

(4) The orthantwise closure of the tropicalization of the L-points of $S$ :

$$
\bigcup_{\sigma \in\{+,-, 0\}^{n}} \overline{\operatorname{trop}_{r}\left(S_{L}^{\sigma}\right) \cap \mathbb{R}^{\sigma} .}
$$

If $S$ is closed, then all these agree with the closure of $\operatorname{trop}_{r}\left(S_{L}\right)$ in $\mathbb{R}^{n}$.

We will denote by $\operatorname{Trop}_{r}(S)$ the set described in the theorem above. The descriptions (2) and (4) are the most commonly found in the literature Ale13, AGS16.

In (4), if $S$ is not closed, we need to take the closure in each orthant separately. For example, for $S=K \backslash\{0\}$, we have $\operatorname{Trop}_{r}(S)=\mathbb{R} \backslash\{0\}$, which is not closed in $\mathbb{R}$ but its intersection with $\mathbb{R}_{>0}, \mathbb{R}_{<0}$, and $\{0\}$ are all closed. In (3) it is necessary to deal with each orthant separately, as we will see in Remark 6.11

For $S$ in the positive orthant defined over $\mathbb{R}$, Alessandrini showed that the logarithm of $\operatorname{Trop}_{r}(S)$ agrees with the logarithmic limit set of $S$ [Ale13, Theorem 4.2], and that $S$ has a description whose tropicalization characterizes $\operatorname{Trop}_{r}(S)$ [Ale13, Corollary 6.7], where inequalities can be joined by both and and or. But in description (3) of the Theorem above, we use only and in each orthant.

It follows from the equivalence of (2) and (4) that the image of any semialgebraic set under the tropicalization map is closed in each orthant, when the value group of the field $M$ is $\mathbb{R}$. See also [AGS16, Theorem 10].

Proof. Since the map trop $r$ respects orthants in the obvious way and (3) and (4) are constructed orthantwise, to prove the equivalence of (1) - (4), we may assume $S=S^{\sigma}$ for some fixed $\sigma \in\{ \pm 1,0\}^{n}$.

$(1) \subseteq(2):$ Since there is a surjection $\left(S_{M}\right)_{r}^{\text {an }} \rightarrow S_{r}^{\text {an }}$ we have $\operatorname{trop}_{r}\left(\left(S_{M}\right)_{r}^{\text {an }}\right) \supseteq \operatorname{trop}_{r}\left(S_{r}^{\text {an }}\right)$. By Theorem 6.2 we have $\operatorname{trop}_{r}\left(\left(S_{M}\right)_{r}^{\text {an }}\right)=\operatorname{trop}_{r}\left(S_{M}\right)$.

$(2) \subseteq(3)$ : This follows from Section 5.2

(3) $\subseteq$ (4): Without loss of generality, suppose $S \subseteq K_{>0}^{n}$. By Theorem 4 of [AGS16, $T:=\log \left(\operatorname{trop}_{r}\left(S_{L}\right)\right)$ is a finite union of integral $\log |K|$-affine polyhedra. By Lemma 6.7 we have $T=\mathbb{R}^{n} \backslash \cup P_{i}=\cap\left(\mathbb{R}^{n} \backslash P_{i}\right)$ for open integral $\log |K|$-affine polyhedra $P_{i}$. Taking real tropical polynomials $F_{P_{i}}$ as in Lemma 6.7, we find $\overline{\left(\operatorname{trop}_{r}\left(S_{L}\right)\right)}=\exp (T)=$ $\cap\left\{-F_{P_{i}} \geq 0\right\}$. Now the statement follows by taking polynomials $f_{P_{i}}$ over $K$ such that $\operatorname{trop}\left(f_{P_{i}}\right)=F_{P_{i}}$ and $f_{P_{i}} \geq 0$ on $S$ as in Lemma 6.8. Finiteness follows from the finiteness in Lemma 6.7. 
$(4) \subseteq(1)$ : We first prove the statement assuming $K=L$; then $S=S_{L}$.

We have the following inclusions, where all closures are taken within the orthant corresponding to $\sigma$ :

$$
\overline{\operatorname{trop}_{r} S \subseteq \overline{\operatorname{trop}_{r}\left(S_{r}^{\text {an }}\right)}}=\operatorname{trop}_{r}\left(\overline{S_{r}^{\text {an }}}\right) \subseteq \operatorname{trop}_{r}(\bar{S}) \subseteq \operatorname{trop}_{r}(S) \subseteq \operatorname{trop}_{r}\left(S_{r}^{\text {an }}\right) .
$$

The first and last inclusion follow from the inclusion $S \subseteq S_{r}^{\text {an }}$. The equality follows because the map trop ${ }_{r}:\left(\mathbb{A}_{r}^{n, \text { an }}\right)^{\sigma} \rightarrow \mathbb{R}^{\sigma}$ is proper by Lemma 3.7, so in particular closed. The next inclusion $\operatorname{trop}_{r}\left(\overline{S_{r}^{\text {an }}}\right) \subseteq \operatorname{trop}_{r}(\bar{S})$ is $(1) \subseteq(4)$, applied to $\bar{S}$. Note here that $(\bar{S})_{r}^{\text {an }}=\overline{S_{r}^{\text {an }}}$. Lastly the inclusion $\operatorname{trop}_{r}(\bar{S}) \subseteq \operatorname{trop}_{r}(S)$ is Lemma 6.4

Now we have to prove the general case, knowing already $\overline{\operatorname{trop}_{r}\left(S_{L}\right)} \subseteq \operatorname{trop}_{r}\left(\left(S_{L}\right)_{r}^{\text {an }}\right)$. Noting that obviously $\left(S_{L}\right)_{M}=S_{M}$ we find

$$
\operatorname{trop}_{r}\left(\left(S_{L}\right)_{r}^{\text {an }}\right) \subseteq \operatorname{trop}_{r}\left(S_{M}\right) \subseteq \operatorname{trop}_{r}\left(S_{r}^{\text {an }}\right) .
$$

Here the first inclusion is $(1) \subseteq(2)$ applied to $S_{L}$ and the second inclusion comes from the fact that the canonical map $S_{M} \rightarrow S_{r}^{\text {an }}$ is compatible with the tropicalization. This proves $(4) \subseteq(1)$ and completes the proof of the equality of $(1)-(4)$.

For the last sentence in the theorem, observe that if $S$ is closed, then $S_{r}^{\text {an }}$ is closed. Then $\operatorname{trop}_{r}\left(S_{r}^{\text {an }}\right)$ is closed by properness of the map trop ${ }_{r}: \mathbb{A}_{r}^{n \text {,an }} \rightarrow \mathbb{R}^{n}$ and the claim follows from the Weak Density Theorem 6.1 and the inclusion $\operatorname{trop}_{r}\left(S_{L}\right) \subseteq \operatorname{trop}_{r}\left(S_{r}^{\mathrm{an}}\right)$.

We have the following Corollary, using Theorem 4.4

Corollary 6.10. If $S$ is semialgebraically connected, then $\operatorname{Trop}_{r}(S)$ is connected.

Remark 6.11. In the description (3) above, it is necessary to consider each orthant separately if $n \geq 2$. That is, it cannot be replaced by

$$
\bigcap_{f \geq 0 \text { on } S}\{\operatorname{trop}(f) \geq 0\} \text {. }
$$

To see this, let $S$ be the complement of the positive orthant in $K^{n}$. We claim that for a polynomial $f$, if $S \subseteq\{f \geq 0\}$, then $\{\operatorname{trop}(f) \geq 0\}=\mathbb{R} \mathbb{T}^{n}$; therefore the intersection above is all of $\mathbb{R}^{n}$.

Let $F$ be a tropical polynomial such that $\{F<0\} \subseteq \mathbb{R}_{>}^{n}$. We will show that $\{F<0\}=\varnothing$, so $\{F \geq 0\}=\mathbb{R} \mathbb{T}^{n}$. Suppose not. Then there exists $y \in\{F<0\}$ where the maximum absolute value among terms in $F(y)$ is attained uniquely at a term

$$
c y_{1}^{a_{1}} y_{2}^{a_{2}} \cdots y_{n}^{a_{n}}<0 \text {. }
$$

Since $\{F<0\}$ is contained in the positive orthant, we have

$$
\begin{array}{r}
c\left(-y_{1}\right)^{a_{1}} y_{2}^{a_{2}} \cdots y_{n}^{a_{n}}>0 \\
c y_{1}^{a_{1}}\left(-y_{2}\right)^{a_{2}} \cdots y_{n}^{a_{n}}>0 \\
c\left(-y_{1}\right)^{a_{1}}\left(-y_{2}\right)^{a_{2}} \cdots y_{n}^{a_{n}}>0,
\end{array}
$$

which is a contradiction since the product of the left hand sides of the four inequalities above must be positive.

Proposition 6.12. Let $S$ be a semialgebraic set of the form $S=\bigcap\left\{f_{i} \leq 0\right\}$. Then we have the following chain of inclusions

$$
\operatorname{int}\left(\operatorname{Trop}_{r}(S)\right) \subseteq \operatorname{int}\left(\bigcap\left\{\operatorname{trop}_{r}\left(f_{i}\right) \leq 0\right\}\right) \subseteq \operatorname{Trop}_{r}(S) \subseteq \bigcap\left\{\operatorname{trop}_{r}\left(f_{i}\right) \leq 0\right\},
$$



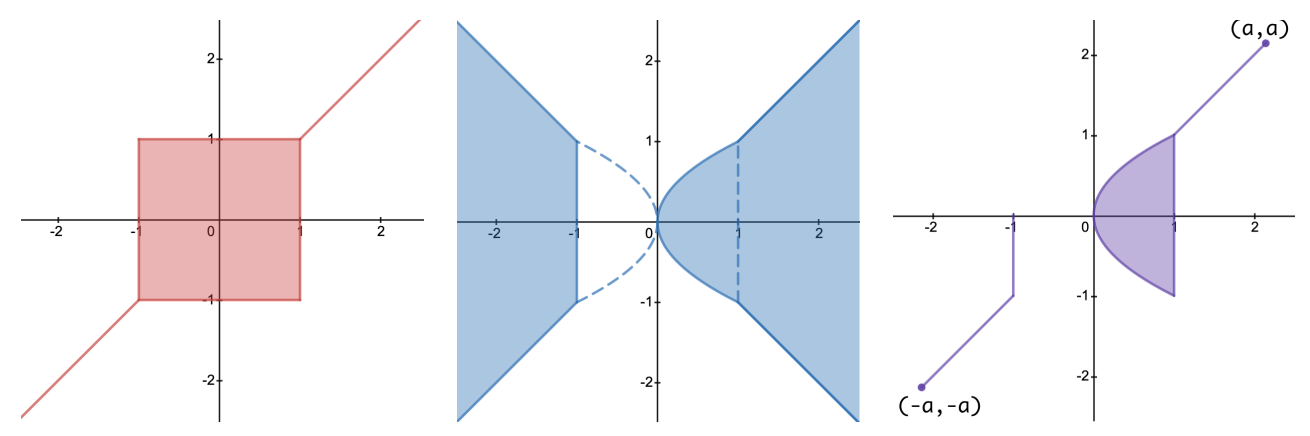

Figure 3. The left and middle figures show the tropicalizations of the sets $\left\{1-(x-y)^{2} \geq 0\right\}$ and $\left\{2 x^{2}+2 x-x y+c y^{2} \geq 0\right\}$ respectively, where $|c|^{\text {sgn }}=-1$. The figure on the right shows the tropicalization of the intersection where $|c|^{\text {sgn }}=-1, c<-1$ and $a=-\frac{1}{|c+1|^{\text {sgn }}}$. See Example 6.13 .

where int denotes the interior.

This Proposition has two consequences for computing the tropicalization of $S=\bigcap\left\{f_{i} \leq\right.$ $0\}$. Firstly, while one may get too big of a set by simply tropicalizing all inequalities defining $S$, the difference will have empty interior. Secondly, if $\bigcap\left\{\operatorname{trop}_{r}\left(f_{i}\right) \leq 0\right\}$ is the

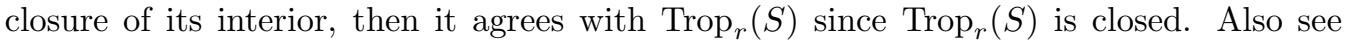
Corollary 16 of AGS16.

Proof. The last inclusion follows from the Fundamental Theorem 6.9 The first inclusion follows from the last one by taking interiors. For the middle inclusion, we first note that

$$
\left(\bigcap\left\{\operatorname{trop}_{r}\left(f_{i}\right) \leq 0\right\}\right) \backslash\left(\bigcap\left(\left\{\operatorname{trop}_{r}\left(f_{i}\right)<0\right\}\right)\right) \subseteq \bigcup\left\{0 \in \operatorname{trop}_{r}\left(f_{i}\right)\right\} .
$$

The last set is contained in an algebraic subset of $\mathbb{R}^{n}$, hence has empty interior. We conclude that

$$
\operatorname{int}\left(\bigcap\left\{\operatorname{trop}_{r}\left(f_{i}\right) \leq 0\right\}\right) \backslash \overline{\bigcap\left\{\operatorname{trop}_{r}\left(f_{i}\right)<0\right\}}=\varnothing
$$

that is, $\operatorname{int}\left(\bigcap\left\{\operatorname{trop}_{r}\left(f_{i}\right) \leq 0\right\}\right) \subseteq \overline{\bigcap\left\{\operatorname{trop}_{r}\left(f_{i}\right)<0\right\}}$. The last set is however contained in $\operatorname{Trop}_{r}(S)$ by the facts that $\operatorname{trop}_{r}(f)\left(|z|^{\mathrm{sgn}}\right)>0$ implies $f(z)>0$ and that $\operatorname{Trop}_{r}(S)$ is closed since $S$ is closed.

Example 6.13. Let $f(x, y)=1-(x-y)^{2}, g_{c}(x, y)=2 x^{2}+2 x-x y+c y^{2}$. For all values of $c$ with $|c|^{\mathrm{sgn}}=-1$, the tropicalization $\operatorname{Trop}_{r}\left(\left\{g_{c} \geq 0\right\}\right)$ is the same, but the tropicalization of the intersection $\{f \geq 0\} \cap\left\{g_{c} \geq 0\right\}$ depends on the choice of $c$. The cases of $c<-1$ with $|c|^{\text {sgn }}=-1$ are depicted in Figure 3. For $-1 \leq c<0$ with $|c|^{\text {sgn }}=-1$, the tropicalization of the intersection contains the two infinite rays as $a \rightarrow \infty$ in the figure. (The dotted curves together with the boundary of the regions form the set $\left\{(X, Y): X^{2}+X-X Y-Y^{2} \ni 0\right\}$. $)$ In any case, difference $\operatorname{Trop}_{r}(\{f \geq 0\}) \cap \operatorname{Trop}_{r}\left(\left\{g_{c} \geq\right.\right.$ $0\}) \backslash \operatorname{Trop}_{r}\left(\{f \geq 0\} \cap\left\{g_{c} \geq 0\right\}\right)$ is a set with empty interior. 
6.3. Limit Theorem. The following is the semialgebraic and real analogue of Payne's theorem in Pay09.

For any finite sequence $\mathcal{F}=\left(f_{1}, \ldots, f_{m}\right)$ in $K\left[x_{1}, \ldots, x_{n}\right]$, let $\operatorname{Trop}_{r, \mathcal{F}}(S) \subseteq \mathbb{R}^{m}$ be the image of the map

$$
\operatorname{trop}_{r, \mathcal{F}}: S_{r}^{\mathrm{an}} \rightarrow \mathbb{R}^{m}, \quad x \mapsto \operatorname{trop}_{r, \mathcal{F}}(x):=\left(\left|f_{1}\right|_{x}^{\operatorname{sgn}}, \ldots,\left|f_{m}\right|_{x}^{\mathrm{sgn}}\right) .
$$

Given a second such sequence $\mathcal{F}^{\prime}=\left(f_{1}^{\prime}, \ldots, f_{r}^{\prime}\right)$ and a map $\tau:\{1, \ldots, m\} \rightarrow\{1, \ldots, r\}$ satisfying $f_{\tau(i)}^{\prime}=f_{i}$, the corresponding projection $\mathbb{R}^{r} \rightarrow \mathbb{R}^{m}, e_{\tau(i)} \mapsto e_{i}$ induces a map $\operatorname{Trop}_{r, \mathcal{F}^{\prime}}(S) \rightarrow \operatorname{Trop}_{r, \mathcal{F}}(S)$. Passing to the limit over all such finite sequences (with respect to maps between the indexing sets), we get the following analogue of Payne's theorem Pay09, Theorem 1.1]:

Theorem 6.14. The canonical map

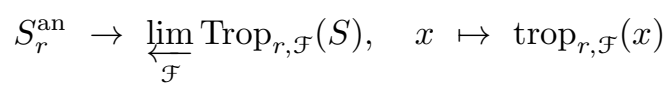

is a homeomorphism, where the limit is taken over the finite families $\mathcal{F}$ in $K\left[x_{1}, \ldots, x_{n}\right]$ as above.

Proof. The map is injective, since if $x \neq y$, there exists a polynomial $f$ with $|f|_{x}^{\mathrm{sgn}} \neq|f|_{y}^{\mathrm{sgn}}$. Consequently, the images of $x$ and $y$ disagree in $\operatorname{Trop}_{r, \mathcal{F}}(S)$ for any $\mathcal{F}$ containing $f$.

To show that the map is surjective, let $z \in \lim _{\mathcal{F}} \operatorname{Trop}_{r, \mathcal{F}}(S)$; it assigns an element $z_{\mathcal{F}} \in$ $\operatorname{Trop}_{r, \mathcal{F}}(S)$ to every finite family $\mathcal{F}$ of polynomials, satisfying compatibility conditions. Define a signed seminorm $|\cdot|_{x}^{\mathrm{sgn}}$ as follows. For $f \in K\left[x_{1}, \ldots, x_{n}\right]$, let

$$
|f|_{x}^{\text {sgn }}:=\left(z_{\mathcal{F}}\right)_{1}
$$

the first coordinate of $z_{\mathcal{F}}$, where $\mathcal{F}=\left(f, f_{2}, \ldots\right)$ is any family whose first component is $f$. This is well-defined since $z_{\mathcal{F}}$ are compatible with maps between the indexing sets as described above.

To see that $|\cdot|_{x}^{\mid s g n}$ is a seminorm, we need to check the following, for all polynomials $f$ and $g$ :

(1) $|f|_{x}^{\operatorname{sgn}}=\operatorname{sgn}(f)|f|_{K}$ if $f \in K$,

(2) $|f \cdot g|_{x}^{\mathrm{sgn}}=|f|_{x}^{\mathrm{sgn}} \cdot|g|_{x}^{\mathrm{sgn}}$

(3) $\min \left(|f|_{x}^{\operatorname{sgn}},|g|_{x}^{\operatorname{sgn}}\right) \leq|f+g|_{x}^{\operatorname{sgn}} \leq \max \left(|f|_{x}^{\mid \operatorname{sgn}},|g|_{x}^{\operatorname{sgn}}\right)$.

Take the tuple $\left(\left(z_{\mathcal{F}}\right)_{1}, \ldots,\left(z_{\mathcal{F}}\right)_{4}\right)$ corresponding to the family $\mathcal{F}=(f, g, f+g, f g)$, and let $y \in S_{r}^{\text {an }}$ be such that $\operatorname{trop}_{r, \mathcal{F}}(y)=\left(\left(z_{\mathcal{F}}\right)_{1}, \ldots,\left(z_{\mathcal{F}}\right)_{4}\right)$. Then $|f|_{x}^{\mathrm{sgn}}=|f|_{y}^{\mathrm{sgn}},|g|_{x}^{\mathrm{sgn}}=|g|_{y}^{\mathrm{sgn}}$, $|f+g|_{x}^{\mathrm{sgn}}=|f+g|_{y}^{\mathrm{sgn}}$ and $|f g|_{x}^{\mathrm{sgn}}=|f \cdot g|_{y}^{\mathrm{sgn}}$, and the axioms (1), (2) and (3) hold for $|\cdot|_{x}^{\operatorname{sgn}}$ because they hold for $|\cdot|_{y}^{\mathrm{sgn}}$.

It remains to show that $|\cdot|_{x}^{\operatorname{sgn}} \in S_{r}^{\text {an }}$. Let us write $S=S_{1} \cup \cdots S_{n}$ as a union of basic semialgebraic subsets. This means that each $S_{i}$ is defined by finitely many inequalities $f_{i j} \geq 0$ and $g_{i k}>0$ where $j=1, \ldots, M_{i}, k=1, \ldots, N_{i}$. Let $\mathcal{F}$ be the family consisting all $f_{i j}$ and $g_{i k}$ and consider $z_{\mathcal{F}}$. Let $y=|\cdot|_{y}^{\operatorname{sgn}} \in S_{r}^{\text {an }} \operatorname{such}_{\text {that }} \operatorname{trop}_{r, \mathcal{F}}(y)=z_{\mathcal{F}}$. Then $|\cdot|_{y}^{\text {sgn }} \in\left(S_{\ell}\right)_{r}^{\text {an }}$ for some $\ell$, and it satisfies all the inequalities $\left|f_{\ell j}\right|_{y}^{\text {sgn }} \geq 0$ and $\left|g_{\ell j}\right|_{y}^{\text {sgn }}>0$. Since we have $\left|f_{\ell j}\right|_{x}^{\text {sgn }}=\left(z_{\mathcal{F}}\right)_{\ell j}=\left|f_{\ell j}\right|_{y}^{\text {sgn }}$ and similarly for the $g_{\ell j}$, we see that $|\cdot|_{x}^{\text {sgn }} \in$ $\left(S_{\ell}\right)_{r}^{\text {an }} \subseteq S_{r}^{\text {an }}$. 
Finally the map is a homeomorphism because the topology on the left is defined as the coarsest topology such that $x \mapsto|f|_{x}^{\text {sgn }}$ is continuous for all $f$, while on the right the topology is defined such that all projection maps, i.e. all maps $\lim _{\mathcal{F}} \operatorname{Trop}_{r, \mathcal{F}}(S) \rightarrow$ $\operatorname{Trop}_{r, \mathcal{F}^{\prime}}(S)$ to a particular $\mathcal{F}^{\prime}$ are continuous. These conditions are equivalent.

\section{ACKNOWLEDGEMENTS}

This work started when CS and JY were in residence at the Mathematical Sciences Research Institute in Berkeley, California, during the Fall 2017 semester on Geometric and Topological Combinatorics, supported by the National Science Foundation under Grant No. DMS-1440140. PJ and JY are grateful to the Institut Mittag-Leffler in Djursholm, Sweden, for hospitality during the Spring 2018 semester. JY thanks the Institute for Computational and Experimental Research in Mathematics in Providence, Rhode Island, for support during the Fall 2018 semester. We thank the referees for helpful comments.

\section{REFERENCES}

[AGS16] Xavier Allamigeon, Stéphane Gaubert, and Mateusz Skomra, Tropical spectrahedra, arXiv:1610.06746 2016.

[Ale13] Daniele Alessandrini, Logarithmic limit sets of real semi-algebraic sets, Adv. Geom. 13 (2013), no. 1, 155-190.

[Bak08] Matthew Baker, An introduction to Berkovich analytic spaces and non-Archimedean potential theory on curves, p-adic geometry, Univ. Lecture Ser., vol. 45, Amer. Math. Soc., Providence, RI, 2008, pp. 123-174.

[BCR98] Jacek Bochnak, Michel Coste, and Marie-Françoise Roy, Real algebraic geometry, Ergebnisse der Mathematik und ihrer Grenzgebiete (3) [Results in Mathematics and Related Areas (3)], vol. 36, Springer-Verlag, Berlin, 1998, Translated from the 1987 French original, Revised by the authors.

[Ber90] Vladimir G. Berkovich, Spectral theory and analytic geometry over non-Archimedean fields, Mathematical Surveys and Monographs, vol. 33, American Mathematical Society, Providence, RI, 1990.

[BHV19] Danielle A. Brake, Jonathan D. Hauenstein, and Cynthia Vinzant, Computing complex and real tropical curves using monodromy, J. Pure Appl. Algebra 223 (2019), no. 12, 5232-5250.

[CD83] Gregory Cherlin and Max A. Dickmann, Real closed rings. II. Model theory, Ann. Pure Appl. Logic 25 (1983), no. 3, 213-231.

[Cel18] Marcel Celaya, The real bergman fan of an oriented matroid, preprint, 2018. Available on the author's web page.

[CR82] Michel Coste and Marie-Françoise Roy, La topologie du spectre réel, Ordered fields and real algebraic geometry (San Francisco, Calif., 1981), Contemp. Math., vol. 8, Amer. Math. Soc., Providence, R.I., 1982, pp. 27-59.

[DY07] Mike Develin and Josephine Yu, Tropical polytopes and cellular resolutions, Experiment. Math. 16 (2007), no. 3, 277-291.

[Dra08] Jan Draisma, A tropical approach to secant dimensions, J. Pure Appl. Algebra 212 (2008), no. 2, 349-363.

[EKL06] Manfred Einsiedler, Mikhail Kapranov, and Douglas Lind, Non-Archimedean amoebas and tropical varieties, J. Reine Angew. Math. 601 (2006), 139-157.

[Jun17] Jaiung Jun, Geometry of hyperfields, arXiv:1707.09348, 2017.

[KMM09] Eric Katz, Hannah Markwig, and Thomas Markwig, The tropical j-invariant, LMS J. Comput. Math. 12 (2009), 275-294.

[KS89] Manfred Knebusch and Claus Scheiderer, Einführung in die reelle Algebra, Vieweg Studium: Aufbaukurs Mathematik [Vieweg Studies: Mathematics Course], vol. 63, Friedr. Vieweg \& Sohn, Braunschweig, 1989 .

[MS15] Diane Maclagan and Bernd Sturmfels, Introduction to tropical geometry, Graduate Studies in Mathematics, vol. 161, American Mathematical Society, Providence, RI, 2015. 
[Pay09] Sam Payne, Analytification is the limit of all tropicalizations, Math. Res. Lett. 16 (2009), no. $3,543-556$.

[Vir84] Oleg Viro, Gluing of plane real algebraic curves and constructions of curves of degrees 6 and 7, Topology (Leningrad, 1982), Lecture Notes in Math., vol. 1060, Springer, Berlin, 1984, pp. 187-200.

[Vir10] Oleg Viro, Hyperfields for tropical geometry I. Hyperfields and dequantization, arXiv:1006.3034. 2010.

[Voj07] Paul Vojta, Nagata's embedding theorem, arXiv:0706.1907, 2007.

Universität Regensburg, 93040 Regensburg, Germany

E-mail address: philipp.jell@ur.de

Fachbereich Mathematik und Statistik, Universität Konstanz, 78457 Konstanz, Germany

E-mail address: claus.scheiderer@uni-konstanz.de

School of Mathematics, Georgia Tech, Atlanta Ga 30332, USA

E-mail address: jyu@math.gatech.edu 